\title{
Canonical Linear Transformation on Fock Space with an Indefinite Metric
}

By

Keiichi R. ITO*

\begin{abstract}
Canonical fields $\Phi_{\varphi}(f)$ on the Fock space with an indefinite metric $<,>=(, \Theta)$ and their canonical linear transformations (Bogolyubov transformations) are investigated.

Let $T$ be a bijective real linear operator preserving the $\varphi$-symplectic form $\left\langle, J>_{r}=\right.$ Real $(, \varphi J)$ in one particle Hilbert space $\mathscr{H}$, where $\varphi$ is unitary and hermitian and $J=$ $\sqrt{-1}$. It is shown that, under some conditions, $T$ has a decomposition $T=V_{1} S V_{2}$, where $V_{\text {, }}$ are $\varphi$-unitary and $S$ is a generalized $\varphi$-scaling, namely $S^{(\varphi)} \equiv \varphi S^{*} \varphi=S, J S J^{-1}=S^{-1}, S K \subset K$ and $S J K \subset J K$ for a decomposition $\mathscr{H}=K \oplus J K$.

$T$ is called $\Theta$-unitarily implementable if there exists a $\Theta$-unitary (bounded bijective $\Theta$ isometric) operator $U_{T}$ on the Fock space $\mathscr{F}$ such that $U_{T} \Phi_{\varphi}(f) U_{T}^{-1}=\Phi_{\varphi}(T f)$. This definition is too restrictive. It is shown that $T$ is $\Theta$-unitarily implementable if and only if $[T, \varphi]$ $=0$ and anti-linear part $T$ - of $T$ is of Hilbert-Schmidt class.

We introduce a less restrictive notion : $T$ is called weakly $\Theta$-unitarily implementable if there exist a $\Theta$-isometric operator $U_{T}^{-1}$ (not necessarily bounded) and a cyclic vector $\Omega_{T} \in \mathscr{F}$ such that $U_{T}^{-1} \Phi_{\varphi}\left(T f_{1}\right) \ldots \Phi_{\varphi}\left(T f_{n}\right) \Omega=\Phi_{\varphi}\left(f_{1}\right) \ldots \Phi_{\varphi}\left(f_{n}\right) \Omega_{T}$, where $\Omega$ is the Fock vacuum. A necessary and a sufficient condition for this implementability are obtained.

As an application, a mass-shift model of the vector field of an indefinite metric formalism (Stückelberg formalism) is discussed. A time-evolution of the system by the model Hamiltonian is investigated.
\end{abstract}

\section{§. Introduction}

Let $\mathscr{L}$ be a Hilbert space equipped with usual (i. e. positive definite) hermitian inner product (.,.), and let $\eta$ be a unitary and hermitian operator: $\eta^{*}=\eta^{-1}=\eta$. We define a new (indefinite) sesquilinear form $\langle.,.\rangle \equiv\left(., \eta_{\bullet}\right)$ on $\mathscr{L}$. This is a "Hlibert space equipped with an indefinite inner product $\langle$,$\rangle ," and denoted \{\mathscr{L},\langle\rangle$,$\} .$

One familiar example of this space is our Minkowski space $M=$ $\left\{\mathscr{L}=R^{4}, \eta=\operatorname{diag}(1,-1,-1,-1)\right\}$. Historically speaking, Pontrya gin

Communicated by H. Araki, November 15, 1977.

* Research Institute for Mathematical Sciences, Kyoto University, Kyoto 606, Japan. 
first investigated this space in order to study differential equations, and physicist also investigated this space in order to describe quantum electrodynamics (QED).

Some examples of indefinite metric formalism in physics are:

(1) An indefinite metric is needed to describe a massless vector field (photon field) in a manifestly covariant way.

(2) In the Stückelberg formalism of the massive vector field, an indefinite metric is used to cancel divergences due to $p^{\mu} p^{\nu} / \mu^{2}$ in the propagator of the Proca field (=vector field of positive metric formalism).

In these examples, a Fock space with an indefinite metric $\langle\rangle=$, $(, \Theta)$ is constructed by the usual tensor algebra constructions from the (one-particle) Hilbert sapce $\mathscr{H}$ with indefinite metric $(, \varphi)$. In this space, fields are defined in terms of creation annihilation operators in a similar manner as the definite metric case.

Real linear transformations on $\mathscr{H}$ which preserve the commutation relations of these fields are called $\varphi$-symplectic transformations and the corresponding transformations of fields are called Bogolyubov transformations.

In this paper, we study the implementability of such Bogolyubov transformations $B$ by linear transformation $U_{B}$ preserving the indefinite metric $(, \Theta)$ in the Fock space.

Our main results are about three different kinds of implementability :

Definition 0-1. $B$ is said to be $\Theta$-unitarily implementable if $U_{B}$ and its inverse are bounded in addition to being $\Theta$-isometric.

We shall show (Theorem 11) that $B$ is $\Theta$-unitarily implementable if and only if $U_{B}$ is unitary with respect to the definite inner product which we use to give the topology for the Fock space. This shows that the restriction of bounded $U_{B}$ (and $U_{B}^{-1}$ ) is too restrictive for our purpose.

Definition 0-2. $B$ is said to be weakly $\Theta$-unitarily implementable 
if the Fock vacuum state is transformed by $B$ to a state given by a cyclic vector in the original Fock space.

This includes a wider class of $B$ compared with Definition $0-1$, and $U_{B}$ may be unbounded though it will preserve the indefinite sesquilinear form in the Fock space. We obtain some conditions on $B$ which are necessary or sufficient for the implementability (Theorems 12, 13). However it is shown that this notion is not invariant under $\varphi$-unitary transformations (bijective linear transformations preserving $(, \varphi))$ of the space $\mathscr{H}$. We introduce a weaker notion called $\Theta$-unitary quasi-implementability, which is invariant under $\varphi$-unitary transformations. For this purpose, we study a decomposition of canonical linear transformations $B:$ A canonical linear transformation $B$ is a bijective real linear transformations of $\mathscr{H}$, which preserves the symplectic form given by the imaginary part of the indefinite inner product $\langle,\rangle \equiv(, \varphi)$ on $\mathscr{H}$.

Under some conditions, such $B$ has the following decomposition:

$$
B=V_{1} S V_{2}
$$

where $V_{1}$ and $V_{2}$ are $\varphi$-unitary and $S$ is a generalized $\varphi$-scaling in the sense that $S$ is a $\varphi$-selfadjoint canonical linear transformation commuting with $C$ for some fixed complex conjugation operator (Theorems 9, 10).

Definition 0-3. A canonical linear transformation $B$ is said to be $\Theta$-unitarily quasi-implementable if $S$-1 is of Hilbert-Schmidt class and its eigenvalues $\lambda$ satisfy

$$
\infty>\Pi_{\lambda} \frac{\left|\lambda+\lambda^{-1}\right|}{2}>0
$$

It is shown that $B$ is $\Theta$-unitarily quasi-implementable in cases Definitions $0-1$ and $0-2$ and that the $\Theta$-unitary quasi-implementability is invariant under $\varphi$-unitary transformations. The $\Theta$-unitary quasiimplementability is shown to be equivalent to the requirement that a kind of non-zero finite inner product between the Fock vacuum 
state and its transformed state can be defined in a certain sense. It is shown, however, that there exists an example of $\Theta$-unitary quasiimplementable $B$ for which the cyclic space for the transformed vacuum has no intersection with the original Fock space.

The organization of this paper is as follows: In $\S 2$, we define a Hilbert space equipped with an indefinite metric, and construct a Fock space with an indefinite metric. In $\S 3$, we study a $\varphi$-symplectic transformation which is a bijective real linear transformation preserving the CCR.

In $\S 4-\S 6$, we consider polar and spectral resolutions of a $\varphi$ symplectic transformation. In $\S 7-\S 10$, the implementability is discussed. Examples are in $\S 11$.

An application is discussed in $\S 12$, where amass-shift model of the vector field of an indefinite metric formalism (Stückelberg formalism) is investigated. The time-evolution by the $\Theta$-selfadjoint Hamiltonian is also discussed.

Concluding remarks are in $\S 13$.

\section{§2. Fock Space with an Indefinite Metric}

In this section we define notation for the Fock space with an indefinite metric. Let $\mathscr{L}_{i}(i=+,-)$ be a Hilbert space with (positive definite hermitian) inner product $(,)_{i}$, and let $\mathscr{L}=\mathscr{L}_{+} \oplus \mathscr{L}_{-}$ be a Hilbert space with inner product $()=,\Sigma_{i}(,)_{2}$.

Let $P_{i}$ be projection operator to $\mathscr{L}_{i}$, and let

$$
\eta=P_{+}-P_{-}
$$

We consider an indefinite hermitian inner product

$$
\langle,\rangle \equiv(, \eta),
$$

and we call the pair $\{\mathscr{L},\langle\rangle$,$\} "a Hilbert space with an indefinite$ metric". See Refs. [4, 16-18,21].

The set of bounded linear operators $C: \mathscr{L}_{i} \rightarrow \mathscr{L}$, is denoted by $\mathscr{B}\left(\mathscr{L}_{i}, \mathscr{L}_{j}\right)$, and $\mathscr{B}(\mathscr{L}, \mathscr{L})$ by $\mathscr{B}(\mathscr{L})$.

Any $A \in \mathscr{B}(\mathscr{L})$ is decomposed as 


$$
\left(\begin{array}{ll}
\mathrm{A}_{++} & A_{+-} \\
A_{-+} & A_{--}
\end{array}\right)
$$

on $\mathscr{L}=\mathscr{L}_{+} \oplus \mathscr{L}$ - where

$$
A_{i j}=P_{i} A P_{j} \in \mathscr{B}\left(\mathscr{L}_{i}, \mathscr{L}_{j}\right) .
$$

Its $\eta$-adjoint $A^{(\eta)} \in \mathscr{B}(\mathscr{L})$ is uniquely defined by

$$
\langle\phi, A \psi\rangle=\left\langle A^{(n)} \phi, \phi\right\rangle .
$$

It is given by

$$
A^{(\eta)}=\eta A^{*} \eta=\left(\begin{array}{rr}
A_{++}^{*} & -A_{-+}^{*} \\
-A_{+-}^{*} & A_{--}^{*}
\end{array}\right) .
$$

Definition 1. $A \in \mathscr{B}(\mathscr{L})$ is said to be

(1) $\eta$-selfadjoint if $A=A^{(\eta)}$,

(2) $\eta$-unitary if $A^{-1}=A^{(\eta)}$.

Remark 1. Even if an operator preserves $\langle$, $\rangle$, it is not necessarily a bounded operator. Note that our definition of an $\eta$-unitary operator requires the boundedness. In this case, if $U$ is $\eta$-unitary, then

$$
\left\|U^{-1}|=|\left|\eta U^{*} \eta !\right|=\right\| U \| .
$$

This also means $1 \leqq \| U_{i} \mid$, and it is easily confirmed that $\|U\|=1$ if and only if $U$ commutes with $\eta$.

We want to introduce a Fock space $\mathscr{F}$ over a Hilbert sapce $\mathscr{H}$ with an indefinite metric $(, \varphi)$. The space $\mathscr{F}$ is defined by

$$
\begin{gathered}
\bigoplus_{n=0}^{\infty} \mathscr{F}(n), \\
\mathscr{F}^{(0)}=C, \mathscr{F}^{(n)}=S_{n}\left[\bigotimes_{n} \mathscr{H}\right] \equiv \bigotimes_{n} \mathscr{H} .
\end{gathered}
$$

Here $S_{n}$ is the following symmetrization operator:

$$
S_{n}\left[\psi_{1} \otimes \cdots \otimes \phi_{n}\right]=(n !)^{-1} \sum_{p e r m} \psi_{\pi(1)} \otimes \cdots \otimes \psi_{\pi(n)} .
$$

Definition 2. For $A \in \mathscr{B}(\mathscr{H}), \Gamma(A)$ is defined by

$$
\Gamma(A) \uparrow \mathscr{F}^{(n)}=A \otimes \cdots \otimes A \quad \text { (n-times), }
$$


and $d \Gamma(A)$ by

$$
d \Gamma(A) \Re^{(n)}=A \otimes 1 \otimes \cdots \otimes 1+\cdots+1 \otimes \cdots \otimes 1 \otimes A .
$$

Their domains of definition are extended by linearity and closure.

Remark 2. $\Gamma(A)$ is a bounded operator if and only if $\|A\| \leqq 1$, and $d \Gamma(A)$ is unbounded whenever $A \neq 0$.

The Fock space $\mathscr{F}$ has a positive definite hermitian inner product $($,$) naturally constructed from ($,$) in \mathscr{H}$. We now introduce on $\mathscr{F}$ the following indefinite inner product

$$
\langle,\rangle=(, \Theta), \Theta=\Gamma(\varphi) \text {. }
$$

Note that $\Theta$ is unitary and hermitian.

The usual creation operator $a^{*}(f)$ for $f \in \mathscr{H}$ is defined by

$$
a^{*}(f)\left(S_{n}\left[\phi_{1} \cdots \otimes \phi_{n}\right]\right)=(\mathrm{n}+1)^{1 / 2} S_{n+1}\left[f \otimes \phi_{1} \otimes \cdots \otimes \psi_{n}\right],
$$

and the selfadjoint (Segal) field by

$$
\Phi(f)=(2)^{-1 / 2}\left[a^{*}(f)+\left(a^{*}(f)\right)^{*}\right]
$$

Though $a^{*}(f)$ is complex linear for $f \in \mathscr{H}, \Phi(f)$ is not complex linear for $f \in \mathscr{H}$.

The $\Theta$-selfadjoint field is defined by

$$
\Phi_{\varphi}(f)=(2)^{-1 / 2}\left[a^{*}(f)+\left(a^{*}(f)\right)^{(\theta)}\right]^{-}
$$

where the bar denotes the closure. Since $\left(a^{*}(f)\right)^{(\theta)}=\Theta\left(a^{*}(f)\right)^{*} \Theta=$ $\left(a^{*}(\varphi f)\right)^{*}$ by the definition of $\Theta$, we have the following commutation relation :

$$
\left[\Phi_{\varphi}(f), \Phi_{\varphi}(g)\right]=i \operatorname{Im}(f, \varphi g)=i \operatorname{Im}\langle f, g\rangle .
$$

The creation operator $a^{*}(f)$ can be expressed in terms of $\Phi_{\varphi}(f)$ by

$$
a^{*}(f)=(2)^{-1 / 2}\left[\Phi_{\varphi}(f)-i \Phi_{\varphi}(J f)\right]
$$

where $J$ is the multiplication operator of $i=\sqrt{-1}$.

Remark 3. $\Phi_{\varphi}(f)$ is $\Theta$-selfadjoint but not $*$ selfadjoint. Then $\exp \left[i \Phi_{\varphi}(f)\right]$ is unbounded in general. 


\section{§3. Canonical Linear Transformation}

Definition 3. A real linear bounded operator $B$ on $\mathscr{H}$ shall be called a canonical linear transformation if the commutation relation is preserved, namely if

$$
\operatorname{Im}(B f, \varphi B g)=\operatorname{Im}(f, \varphi g) .
$$

A canonical linear transformation $B$ shall be called a $\varphi$-symplectic transformation if $B$ is bijective.

From the definition, it follows that canonical linear transformations form a semi-group, and $\varphi$-symplectic transformations $(=\varphi$-Bogolyubov transformations) a group.

To obtain an operator form of the condition for $B$, we introduce a real bilinear inner product on $\mathscr{H}$ by

$$
(f, g)_{r}=\operatorname{Re}(f, g),
$$

and denote the multiplication of $i$ by $J$. The adjoint of real linear operator $B$ with respect to $(,)_{r}$ will be also denoted by $B^{*}$. It coincides with ordinary $*$ if $B$ is complex linear (namely if $[B, J]$ $=0)$. We define $B^{(\varphi)}=\varphi B^{*} \varphi$.

Lemma 1. $B$ is canonical linear transformation if and only if

$$
B^{(\varphi)} J B=J \text {. }
$$

Proof. (3-1) is equivalent to

$$
(B f, \varphi J B g)_{r}=(f, \varphi J g)_{r},
$$

which is equivalent to

$$
\left(f, \varphi\left[B^{(\varphi)} J B-J g\right)_{r}=0\right.
$$

for all $f, g \in \mathscr{H}$.

Q. E. D.

Any real linear operator $B$ can be uniquely decomposed as a sum of complex linear and anti-linear operators :

$$
B=B_{+}+B_{-}, B_{ \pm}=\frac{1}{2}\left(B \pm J B J^{-1}\right)
$$


When $B$ is a $\varphi$-symplectic operator, $B_{-}$is called as its "off-diagonal part".

Lemma 2. $B$ is a canonical linear transformation if and only if

$$
\left\{\begin{array}{l}
B_{+}^{(\varphi)} B_{+}-B_{-}^{(\varphi)} B_{-}=1, \\
B_{+}^{(\varphi)} B_{-}=B_{-}^{(\varphi)} B_{+} .
\end{array}\right.
$$

Proof. (3-3) is equivalent to

$$
\left(B_{+}^{(\varphi)} B_{+}-B_{-}^{(\varphi)} B_{-}\right)+\left(B_{+}^{(\varphi)} B_{-}-B_{-}^{(\varphi)} B_{+}\right)=1 .
$$

Complex linear and anti-linear parts of two sides of this equation are the two equations in this lemma.

Q. E. D.

We are interested in the transformation of the field under Bogolyubov transformation, which is given by

$$
\pi_{B}\left(\Phi_{\varphi}(f)\right) \equiv \Phi_{\varphi}^{(B)}(f) \equiv \Phi_{\varphi}(B f) .
$$

\section{§4. Polar Decomposition of $\varphi$-Symplectic Transformation}

The main purpose in this section is to prove the following theorem :

Theorem 3. A $\varphi$-symplectic operator $T$ such that 0 is not an eigenvalue of $T^{(\rho)} T+|T|^{(\varphi)}|T|$ has the following decomposition:

$$
T=O \theta|T|
$$

where $O, \theta$ and $|T|$ are $\varphi$-symplectic operator such that $|T|$ is selfadjoint positive,

(1) $\theta$ is orthogonal and $\varphi$-selfadjoint,

(2) $O$ is complex linear and unitary.

This decomposition is unique up to the transformation

$$
(O, \theta) \rightarrow\left(O V, V^{-1} \theta\right)
$$

by selfadjoint, unitary operator $V$ commuting with $\varphi$.

We prove this theorem through several lemmas $[20,24,27]$. 
Lemma 4. Let $T$ be a $\varphi$-symplectic operator. Then there is a unique decomposition of $T$ as follows:

$$
T=Q|T|
$$

where $|T|$ and $Q$ are $\varphi$-symplectic and

$$
|T|^{*}=|T|>0, Q^{*}=Q^{-1}
$$

Proof. Let

$$
T=Q|T|
$$

be the unique polar decomposition of an invertible operator in a real Hilbert space. Since $T$ is $\varphi$-symplectic, we have

$$
\varphi J T^{*} \varphi J^{-1}=T^{-1}
$$

Hence

$$
\varphi J|T| \varphi J^{-1} \varphi J Q^{*} \varphi J^{-1}=|T|^{-1} Q^{-1} .
$$

By the uniqueness of the decomposition, we have

$$
\varphi J Q J^{-1} \varphi=Q, \varphi J|T| J^{-1} \varphi=|T|^{-1}
$$

Q. E. D.

Lemma 5. Let $Q$ be an orthogonal operator commuting with $\varphi J$. Assume that -1 is not an eigenvalue of $Q^{(\varphi)} Q$. Then there is the following decomposition of $Q$ :

$$
Q=O \theta
$$

where

$$
\left\{\begin{array}{l}
\theta^{*}=\varphi \theta \varphi=J \theta J^{-1}=\theta^{-1} \\
O^{*}=O^{-1}, J O J^{-1}=\varphi O \varphi=O .
\end{array}\right.
$$

This decomposition is unique up to a unitary and hermitian operator commuting with $\varphi$.

Proof. We explicitly construct $\theta=\left(Q^{(\varphi)} Q\right)^{1 / 2}$.

Let

$$
p=\frac{1}{2}(\varphi+1)=P_{+}, \quad p^{\prime}=Q^{*} p Q
$$

be two projection operators. For any two projections $E$ and $F$, we 
define [2]

$$
E \Lambda F=\lim _{n \rightarrow \infty}(E F)^{n} .
$$

We first note that $p^{\prime} \Lambda(1-p)+p \Lambda\left(1-p^{\prime}\right)=0$ due to the absence of the eigenvalue -1 for $Q^{(\varphi)} Q=[p-(1-p)]\left[p^{\prime}-\left(1-p^{\prime}\right)\right]$.

Let

$$
\theta=(1-p) \Lambda\left(1-p^{\prime}\right)+p \Lambda p^{\prime}+\Delta\left(1-p \Lambda p^{\prime}-(1-p) \Lambda\left(1-p^{\prime}\right)\right)
$$

where

$$
\begin{aligned}
\Delta=\left(p p^{\prime} p\right)^{1 / 2}+ & {\left[(1-p)\left(1-p^{\prime}\right)(1-p)\right]^{1 / 2}+\left(p p^{\prime} p\right)^{1 / 2} p p^{\prime}(1-p) } \\
- & {\left[(1-p)\left(1-p^{\prime}\right)(1-p)\right]^{1 / 2}(1-p) p^{\prime} p . }
\end{aligned}
$$

Then by the construction [2],

$$
\theta^{*}=\theta^{-1}, J \theta J^{-1}=\varphi \theta \varphi=\theta^{-1}, \theta^{2}=Q^{(\varphi)} Q
$$

Next let

$$
O=Q \theta^{-1}
$$

Then $O$ is again orthogonal and commutes with $\varphi J$. Further $O$ also commutes with $\varphi$ (then also with $J$ ) :

$$
O^{-1} \varphi O=\theta Q^{-1} \varphi Q \theta^{-1}=\theta \varphi \theta^{2} \theta^{-1}=\varphi \text {. }
$$

Q. E. D.

\section{§ 5. Another Decomposition of $f$-Symplectic Transformations}

We first introduce several notions $[16-18,21]$ :

Definition 4. A closed subspace $\mathfrak{S}$ of a Hilbert space $\mathscr{H}$ equipped with an indefintie sesqui-linear form $\langle$,$\rangle is said to be$

(1) non-negative (resp. non-positive) if and only if $\langle x, x\rangle \geqq 0$ (resp. $\leqq 0)$ for any $x \in \mathfrak{S}$,

(2) positive (resp. negative) if and only if $\langle x, x\rangle>0($ resp. $\langle 0)$ for any $x(\neq 0) \in \mathfrak{S}$,

(3) uniformly positive (resp. uniformly negative) if and only if there is a non-zero positive constant $\mu$ such that $\langle x, x\rangle \geqq \mu(x, x) \quad(\operatorname{resp} . \leqq-\mu(x, x))$ for any $x \in \mathfrak{F}$, 
(4) $\varphi$-complementary if and only if

$$
\mathfrak{S}^{<\perp>} \cap \mathfrak{S}=\{0\}
$$

where $\mathfrak{S}^{<\perp>}$ is the orthogonal complement of $\mathfrak{S}$ in $\mathscr{H}$ with respect to $\langle$,$\rangle .$

Definition 5. A $\varphi$-selfadjoint operator $A$ is said to be

(1) non-negative (resp. non-positive) if and only if $\langle x, A x\rangle \geqq 0$ (resp. $\leqq 0)$ for any $x \in \mathscr{H}$,

(2) positive (resp. negative) if and only if $\langle x, A x\rangle>0$ (resp. $\langle 0)$ for any $x(\neq 0) \in \mathscr{H}$,

(3) uniformly positive (resp. uniformly negative) if and only if there is a non-zero positive constant $\mu$ such that $\langle x, A x\rangle \geqq \mu(x, x) \quad($ resp. $\leqq-\mu(x, x))$ for any $x \in \mathscr{H}$.

We study absolute value $|T|$ of a $\varphi$-symplectic operator $T$ on $\mathscr{H}$ relative to the real indefinite inner product $\langle,\rangle_{r}=(, \varphi)_{r}$. Let

$$
H=\varphi|T|
$$

Then this satisfies

$$
H^{(\varphi)}=H, J H J^{-1}=H^{-1} .
$$

Moreover $H$ is uniformly positive with respect to $\langle,\rangle_{r}$ :

$$
\langle x, H x\rangle_{r}=(x,|T| x) \geqq \mu(x, x)
$$

with $\mu=\|\left.|T|^{-1}\right|^{1-1}$.

For a uniformly positive $\eta$-selfadjoint operator $A$ on a complex Hilbert space $\mathscr{H}$ with an indefinite metric $\eta, A$ has the following spectral resolution in terms of $\eta$-selfadjoint projection operators $\{E(\Omega) ; \Omega \subset(-\infty, \infty)\}$ (see for example [16-18]) :

$$
A=\int_{-\infty}^{\infty} \lambda d E(\lambda)
$$

where $E(\Omega)$ is $\sigma$-additive,

$$
\begin{gathered}
E^{(\eta)}(\Omega)=E(\Omega), E(\Omega) E\left(\Omega^{\prime}\right)=E\left(\Omega \cap \Omega^{\prime}\right), \\
E(-\infty, \infty)=1
\end{gathered}
$$


and $E(\Omega)$ is uniquely determined by $A$.

We take the complexification of $\mathscr{H}$ to be $\mathscr{H}_{c}$. Define the complex conjugation operator $C$ on $\mathscr{H}_{c}$ by

$$
C(f \oplus i g)=f \oplus-i g .
$$

We extend $H$ to be an operator on $\mathscr{K}_{c}$ satisfying $C H C=H$. Then $H$ satisfies the requirement for Aabove and hence we have a spectral resolution

$$
H=\int_{-\infty}^{\infty} \lambda d E(\lambda)
$$

From $C H C=C$ and the uniqueness of $E(\Omega)$, we have $C E(\Omega) C$ $=E(\Omega)$ and hence $E(\Omega)$ leaves the real linear subspace $\mathscr{H}$ of $\mathscr{H}_{c}$ invariant. Hence we can restrict the above resolution to $\mathscr{H}$.

Since $J H J^{-1}=H^{-1}$, we also have

$$
J E(\Omega) J^{-1}=E\left(\Omega^{-1}\right) .
$$

Lemma 6. Let $H$ be a $\varphi$-selfadjoint $\varphi$-symplectic operator which is uniformly positive with respect to $\langle,\rangle_{r}$. Then there exists a real subspace $K^{\prime} \subset \mathscr{H}$ such that $K^{\prime} \cap J K^{\prime}=\{0\}, \mathscr{H}=K^{\prime} \oplus J K^{\prime}$ with respect to $\langle,\rangle_{r}$ and

$$
H K^{\prime} \subset K^{\prime}, H\left(J K^{\prime}\right) \subset J K^{\prime}
$$

Proof. We use the spectral resolution (5-7). Let

$$
\mathscr{H}(\{ \pm 1\})=E(\{ \pm 1\}) \mathscr{H} .
$$

Then $J \mathscr{H}(\{ \pm 1\})=\mathscr{H}(\{ \pm 1\})$. Since $\pm<,\rangle_{r} \geqq \mu($,$) on \mathscr{H}(\{ \pm 1\})$, it is possible to find a subspace $I( \pm 1)$ of $\mathscr{H}(\{ \pm 1\})$ satisfying

$$
\begin{aligned}
& I( \pm 1) \cap J I( \pm 1)=\{0\}, \\
& \mathscr{H}(\{ \pm 1\})=I( \pm 1) \oplus J I( \pm 1),
\end{aligned}
$$

where direct sum referes to the orthogonality with respect to $\langle,\rangle_{r}$.

Let

$$
K^{\prime}=E((-1,1)) \mathscr{H} \oplus I(+1) \oplus I(-1)
$$

Then

$$
J K^{\prime}=(E(-\infty,-1)+E(1, \infty)) \mathscr{H} \oplus J I(+1) \oplus J I(-1),
$$

and 


$$
\begin{gathered}
H K^{\prime} \subset K^{\prime}, H\left(J K^{\prime}\right) \subset J K^{\prime}, K^{\prime} \cap J K^{\prime}=\{0\}, \\
\mathscr{H}=K^{\prime} \oplus J K^{\prime} .
\end{gathered}
$$

Q. E. D.

To state the main result of this section, we introduce the follwing terminology :

Definition 6. In a $2 \times 2$ operator entry representation of an operator on $\mathscr{H}$ relative to the given decomposition $\mathscr{H}=K \oplus J K$ where direct sum referes to the orthogonality with respect to $(,)_{r}$ and $\langle,\rangle_{\text {r }}$ an operator

$$
S=\left(\begin{array}{cc}
A & 0 \\
0 & A^{-1}
\end{array}\right)
$$

with $\varphi$-selfadjoint uniformly positive operator $A$ is called a $\varphi$-scaling. (The unique J-linear extension of $A$ to $\mathscr{H}$ will be denoted by the same letter $A$.)

Our discussion above yields the following main result in this section :

Theorem 7. A $\varphi$-symplectic operator $T$, such that the orthogonal part $Q$ in the polar decomposition of $T$ commutes with $\varphi$ (or equivalently commutes with $J$ ), has the decomposition

$$
T=U_{1} S U_{2}
$$

with $\varphi$-unitary $U_{i}$ and a $\varphi$-scaling $S$.

Proof. Let

$$
K^{\prime}=K_{+}^{\prime} \oplus K_{-}^{\prime}
$$

where

$$
K_{+}^{\prime}=E(0,1) \mathscr{H} \oplus I(+1), K_{-}^{\prime}=E(-1,0) \mathscr{H} \oplus I(-1)
$$

Since for $x \in K_{x}^{\prime}$,

$$
(x, x)_{r} \geqq \pm\langle x, x\rangle \geqq\langle x, H x\rangle_{r} \geqq \mu(x, x),
$$

there exists a bijective $\varphi$-isometric (namely $\varphi$-orthogonal) operator $U$ on $\mathscr{H}$ which maps $K$ and $J K$ onto $K^{\prime}$ and $J K^{\prime}$ respectively. Then 
$U$ is $\varphi$-unitary and $T=Q \varphi H=Q \varphi U^{-1} S U_{-1} \equiv U_{1} S U_{2}$.

Q. E. D.

A notion of $\varphi$-scaling is an extension of the notion of scaling operator in symplectic space $[15,27]$. When $\varphi=1$, this decomposition is in [24].

The operator $T$ of Theorem 6 corresponds to the case $\Theta=1$ in Theorem 3. On the other hand, the operator $\theta$ itself has a similar diagonalization as above.

Lemma 8. Let $\theta$ be an orthogonal operator such that

$$
\varphi \theta \varphi=J \theta J^{-1}=\theta^{-1} .
$$

Then there exists a $\theta$-invariant real subspace $K^{\prime \prime}$ such that $K^{\prime \prime} \cap J K^{\prime \prime}$ $=\{0\}, K^{\prime \prime}$ and $J K^{\prime \prime}$ are mutually orthogonal with respect to both $\langle,\rangle_{r}$ and $(,)_{r}$ and

$$
\mathscr{H}=K^{\prime \prime} \oplus J K^{\prime \prime}
$$

In this case

$$
u=\theta \uparrow K^{\prime \prime}, \quad u^{-1}=\theta \uparrow J K^{\prime \prime}
$$

are orthogonal operators such that $\varphi u \varphi=u^{-1}$.

Proof. After the complexification, we have

$$
\theta=\int_{-\pi}^{\pi} e^{i \tau} d P(\tau)
$$

where $\{P(\Omega) ; \Omega \subset[-\pi, \pi]\}$ are selfadjoint projections such that

$$
\left\{\begin{array}{l}
\varphi P(\Omega) \varphi=J P(\Omega) J^{-1}=P(-\Omega) \quad \text { for } \Omega \subset[0, \pi) \\
\varphi[P(-\pi)+P(\pi)] \varphi=J[P(-\pi)+P(\pi)] J^{-1}=P(-\pi)+P(\pi) .
\end{array}\right.
$$

Since $P(\Omega)$ commutes with $\varphi J$ for all $\Omega \subset(0, \pi)$, there exists a decomposition $\mathscr{H}(\Omega)=P(\Omega) \mathscr{H}=k(\Omega) \oplus \varphi J k(\Omega)$ into subspaces $k(\Omega)$ and $\varphi J k(\Omega)$ which are $P(\Omega)$-invariant for all $\Omega: P(\Omega) \mathscr{H}$ is decomposed as

$$
k(\Omega) \oplus \varphi J k(\Omega)
$$

where $k(\Omega) \perp \varphi J k(\Omega)$ with respect to $(,)_{r}$. Let $P_{1}(\Omega)$ and $P_{2}(\Omega)$ be selfadjoint projections to $k(\Omega)$ and $\varphi J k(\Omega)$ respectively. They 
satisfy

$$
P_{2}(\Omega)=\varphi J P_{1}(\Omega) \varphi J^{-1}, P_{1}(\Omega) P_{2}(\Omega)=0
$$

and

$$
P(\Omega)=P_{1}(\Omega)+P_{2}(\Omega)
$$

Define

$$
P_{i}(-\Omega)=\varphi P_{i}(\Omega) \varphi \quad i=1,2 \text {. }
$$

Then $\left\{P_{i}(-\Omega)\right\}$ again satisfy $(5-16)$ and (5-17), and

(5-19) $J P_{1}(\Omega) J^{-1}=P_{2}(-\Omega), J P_{2}(\Omega) J^{-1}=P_{1}(-\Omega) \quad$ for $\quad \Omega \subset(0, \pi)$.

By the construction $P_{i}(\Omega)+P_{i}(-\Omega)$ with $i=1,2$ are selfadjoint projection operators commuting with $\varphi$.

For $\mathscr{H}(1)=P(0) \mathscr{H}$ and $\mathscr{H}(-1)=(P(\pi)+P(-\pi)) \mathscr{H}$, there are similar decompositions as Lemma 6 :

$$
\mathscr{H}( \pm 1)=I_{ \pm} \oplus J I_{ \pm},
$$

where $I_{ \pm}$are orthogonal to $J I_{ \pm}$with respect to both $(,)_{r}$ and $\langle,\rangle_{r}$.

Let

$$
K^{\prime \prime}=\left[P_{1}((-\pi, 0))+P_{1}((0, \pi))\right] \mathscr{H} \oplus I_{+} \oplus I_{-} .
$$

Then

$$
J K^{\prime \prime}=\left[P_{2}((-\pi, 0))+P_{2}((0, \pi))\right] \mathscr{H} \oplus J I_{+} J I_{-}
$$

and

$$
u=\left(\int_{-\pi}^{0}+\int_{0}^{\pi}\right) e^{i \tau} d P_{1}(\tau)+P_{+}-P_{-}
$$

where $P_{ \pm}$are projections to $I_{ \pm}$.

Q. E. D.

\section{§ 6. $\varphi$-Polar Decomposition of $\varphi$-Symplectic Operator}

We shall discuss the polar decomposition of a $\varphi$-symplectic operator relative to the $\varphi$-inner product:

$$
T=U H
$$


where $U$ should be $\varphi$-unitary operator and $H \varphi$-selfadjoint, $\varphi$-symplectic with spectrum in the right half plane. Even in a finite dimensional case, there are examples of $T$ for which such a decomposition is not possible. Therefore we impose a condition in the spectrum of $T$ in the following theorem:

Theorem 9. Let $T$ be $\varphi$-symplectic, $T_{ \pm}=\frac{1}{2}\left(T_{ \pm} J T J^{-1}\right)$ be its complex linear and anti-linear parts. Assume that $T_{-}$is of HilbertSchmidt class and the closed negative real axis $[-\infty, 0]$ belongs to the resolvent set of $T_{+}^{(\varphi)} T_{+}$. Then there exists a $\varphi$-unitary operator $U$ and $\varphi$-selfadjoint $\varphi$-symplectic $H$ with its spectrum in the right half plane satisfying

$$
T=U H \text {. }
$$

Such a pair $(U, H)$ is unique and satisfies

$$
J U=U J, J H J^{-1}=H^{-1} \text {. }
$$

Definition 7. Relative to a given orthogonal and $\varphi$-orthogonal decomposition $\mathscr{H}=K \oplus J K$, a $\varphi$-selfadjoint $\varphi$-symplectic operator $S$ is called a generalized $\varphi$-scaling if $S$ has the form

$$
\left(\begin{array}{ll}
h & 0 \\
0 & h^{-1}
\end{array}\right)
$$

in the $2 \times 2$ matrix representation of an operator on $\mathscr{H}$ relative to the decomposition $\mathscr{H}=K \oplus J K$, where $h$ is a $\varphi$-selfadjoint operator leaving $K$ and $J K$ invariant.

Theorem 10. For an orthogonal and $\varphi$-orthogonal decomposition

$$
\mathscr{H}=K \oplus J K,
$$

assume that a $\varphi$-symplectic operator $T$ leaves $K$ and $J K$ invariant and satisfies the assumption of Theorem 9. Then $T$ has the following unique decomposition:

$$
T=U S
$$

where $U$ is $\varphi$-unitary and $S$ is a generalized $\varphi$-scaling with its spectrum in the right half plane. 
Proof of Theorem 9. Let

$$
A=T^{(\varphi)} T=\left(2 T_{+}^{(\varphi)} T_{+}-1\right)+2 T_{+}^{(\varphi)} T_{-} .
$$

By assumption, $A$ has the following properties:

(1) $A-1$ is compact because $T_{+}^{(\varphi)} T_{+}-1=T_{-}^{(\varphi)} T_{-}$(3-4) is of trace class and $T_{-}$is of Hilbert-Schmidt class.

(2) $A$ does not have a negative or zero eigenvalues. This is an immediate consequence of the assumption about the spectrum of $T_{+}^{(\varphi)} T_{+}$and the identity

$$
\frac{1}{2}\left(A+A^{-1}\right)=2 T_{+}^{(\varphi)} T_{+}-1
$$

which follows from $J A J^{-1}=A^{-1}$ and $J T_{+}^{(\varphi)} T_{-} J^{-1}=-T_{+}^{(\varphi)} T_{-}$.

We construct the operator

$$
H=-(2 \pi i)^{-1} A \int_{\Gamma}(A-z)^{-1} z^{-1 / 2} d z
$$

in the complexification of the real Hilbert space $\left\{\mathscr{H},(,)_{r}\right\}$, where $z^{-1 / 2}$ is defined on the complex plane with the cut on the negative real axis such that $z^{-1 / 2}>0$ for positive real $z$ and contour $\Gamma$ may be taken to be the union of the upper side of the cut from $-\infty$ to 0 and the lower side of the cut from 0 to $-\infty$.

Due to the two properties of $A$, the operator calculas in Chapter 7 of [8] is applicable. By Theorems 10 and 11 in that Chapter, $H_{2}=$ $A$ and the spectrum of $H$ lies in the right half plane. By a straightforward calculation, we have

$$
H=\frac{A}{\pi} \int_{-\infty}^{\infty}\left(A+\tau^{2}\right)^{-1} d \tau
$$

Hence $H$ can be considered as a real linear operator on $\mathscr{H}$. By $\varphi$ selfadjointness of $A$, we have

$$
H^{(\varphi)}=\frac{A^{(\varphi)}}{\pi} \int_{-\infty}^{\infty}\left(A^{(\varphi)}+\tau^{2}\right)^{-1} d \tau=H
$$

Since $A$ is $\varphi$-symplectic,

$$
J H J^{-1}=\frac{A^{-1}}{\pi} \int_{-\infty}^{\infty}\left(A^{-1}+\tau^{2}\right)^{-1} d \tau
$$




$$
=\frac{1}{\pi} \int_{-\infty}^{\infty}\left(A+\sigma^{2}\right)^{-1} d \sigma=A^{-1} H
$$

by the change of variable $\tau=\sigma^{-1}$. Since $A=H^{2}$, we have $J H J^{-1}=$ $H^{-1}$. Hence $H$ is a $\varphi$-selfadjoint, $\varphi$-symplectic operator with its spectrum (on the complexified space) in the right half plane.

Let $U=T H^{-1}$. We have

$$
U^{(\varphi)}=\left(H^{-1}\right)^{(\varphi)} T^{(\varphi)}=H^{-1} T^{(\varphi)} .
$$

Hence

$$
U^{(\varphi)} U=H^{-1} T^{(\varphi)} T H^{-1}=H^{-1}\left(H^{2}\right) H^{-1}=1 .
$$

Since $U$ is invertible, $U$ is $\varphi$-unitary. Since $T^{(\varphi)}$ and $H$ are $\varphi$-symplectic, $U$ is also $\varphi$-symplectic, which implies

$$
J U J^{-1}=\left(U^{(\varphi)}\right)^{-1}=U .
$$

Thus we have the desired decomposition $T=U H$.

To show the uniqueness, let $U_{1} H_{1}$ be another decomposion. Then $H_{1}^{2}=T^{(\varphi)} T=H^{2}$. By the uniqueness of the square-root with the spectrum in the right half plane (see, for example, Chapter 7 in [8]), we have $H_{1}=H$, and hence $U_{1}=U$.

Q. E. D.

Proof of Theorem 10. We define

$$
T \uparrow K=T_{1}, \quad T \uparrow J K=T_{2} .
$$

Since $T$ is $\varphi$-symplectic,

$$
J T_{1}^{-1} J^{-1}=T_{2}^{(\varphi)}
$$

and hence

$$
J T_{1}^{(\varphi)} T_{1} J^{-1}=\left(T_{2}^{(\varphi)} T_{2}\right)^{-1}
$$

Let

$$
h=\left(T_{1}^{(\varphi)} T_{1}\right)^{1 / 2} \oplus J\left(T_{1}^{(\varphi)} T_{1}\right)^{1 / 2} J^{-1},
$$

and let

$$
U=T_{1}\left(T_{1}^{(\varphi)} T_{1}\right)^{-1 / 2} \oplus J T_{1}\left(T_{1}^{(\varphi)} T_{1}\right)^{-1 / 2} J^{-1} .
$$

Then for $f \in J K$

$$
\left(T_{2}^{(\varphi)} T_{2}\right)^{1 / 2} f=h^{-1} f
$$


and

$$
\begin{aligned}
T_{2}\left(h^{-1}\right)^{-1} f & =T_{2} h^{2} h^{-1} f=T_{2}\left(T_{2}^{(\varphi)} T_{2}\right)^{-1} h^{-1} f \\
& =T_{2}^{(\varphi)-1} h^{-1} f=T_{1} h^{-1} f=U f .
\end{aligned}
$$

Q. E. D.

Our question is whether the $\varphi$-selfadjoint, $\varphi$-symplectic operator $H$ is always similar to a generalized $\varphi$-scaling $S$ via suitable $\varphi$-unitary operator $V$ :

$$
H=V S V^{-1} \text {. }
$$

This is affirmative for $\operatorname{dim} \mathscr{H}<\infty$, and $T$ in Theorem 9 always has a decomposition

$$
T=V_{1} S V_{2}
$$

where $S$ is a generalized $\varphi$-scaling with its spectrum in the right half plane and $V_{i}$ are $\varphi$-unitary operators. But for $\operatorname{dim} \mathscr{H}=\infty, \varphi$-isometric operator $V$ seemes to be unbounded in general.

As we have already proved in Theorem 7 and Lemma 8, this diagonalization is always possible if $H$ is uniformly positive or orthogonal.

For given generalized $\varphi$-scaling $S$, let $\varphi$-symplectic operator $T$ be given by $V_{1} S V_{2}$ where $V_{i}$ are $\varphi$-unitary. By the boundedness of $V_{1}$ and $V_{2}$, (H. S. means Hilbert-Schmidt)

$$
\begin{aligned}
T_{-} \text {is H.S. } & \leftrightarrow S-S^{-1} \text { is H.S. } \\
& \leftrightarrow S^{2}-1 \text { is H.S. }
\end{aligned}
$$

The role of condition that $(-\infty, 0]$ is in the resolvent set of $T_{+}^{(\varphi)} T_{+}$in Theorem 9 is necessary by the following example which does not have a $\varphi$-polar decomposition as (6-1):

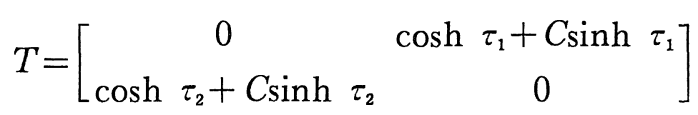

on $\mathscr{H}=C^{2}$ where $\tau_{1} \neq \tau_{2}, \varphi=\operatorname{diag}(1,-1)$ and $C$ denotes the complex conjugation. 


\section{§ 7. $\Theta$-Unitary Implementability}

Recall that a $\varphi$-symplectic operator $T$ is $\Theta$-unitarily implementable if there exists a $\Theta$-unitary operator $U_{T}$ such that $U_{T} \Phi_{\varphi}(f) U_{T}^{-1}=\Phi_{\varphi}(T f)$.

The main result in this section is the following:

Theorem 11. $T$ is $\Theta$-unitarily implementable if and only if $T$ commutes with $\varphi$ and $T_{-}$is in the Hilbert-Schmidt class.

Proof. We first prove that if $T$ does not commute with $\varphi$, then $U_{T}^{-1}$ does not exist. Let $f \in \mathscr{H}_{+}=P_{+} \mathscr{H}$. Then $\Phi_{\varphi}(f)$ is selfadjoint and $\exp \left[i \Phi_{\varphi}(f)\right]$ is a bounded operator with norm one. $U_{\varphi}$ implements

$$
U_{T} \Phi_{\varphi}(f) U_{T}^{-1}=\Phi_{\varphi}(T f)=\Phi_{\varphi}\left(P_{+} T f\right)+\Phi_{\varphi}\left(P_{-} T f\right),
$$

where $\Phi_{\varphi}\left(P_{+} T f\right)$ and $i \Phi_{\varphi}\left(P_{-} T f\right)$ are selfadjoint and commute each other. Thus $\Phi_{\varphi}(T f)$ is a normal operator. If $U_{T}$ is $\Theta$-unitary, then $\left\|U_{T}\right\|=\left\|U_{T}^{-1}\right\|<\infty$ and

$$
U_{T} e^{i \Phi_{\varphi}(f)} U_{T}^{-1}=e^{i \Phi_{\varphi}(P+T f)} e^{i \Phi_{\varphi}(P-T f)}
$$

must be a bounded operator. But $\| \exp \left[i \Phi_{\varphi}\left(P_{-} T f\right) \|=\infty\right.$ whenever $P_{-} T f \neq 0$. Then $P_{-} T P_{+}=0$ if $U_{T}$ is $\Theta$-unitary. Next let $f \in \mathscr{H}_{-}$. Then $i \Phi_{\varphi}(f)$ is selfadjoint in this case. By similar reasons, we see that $P_{+} T P_{-}=0$ if $U_{T}$ is $\Theta$-unitary. Thus $T$ commutes with $\varphi$ whenever $T$ is $\Theta$-unitarily implementable.

We may restrict our attention to the case where $T$ commutes with $\varphi$. We use the decomposition $\mathscr{H}=\mathscr{H}_{+} \oplus \mathscr{H}_{-}$according to the eigenvalue \pm 1 of $\varphi$. Correspondingly we have $T=T_{1} \oplus T_{2}, \mathscr{F}(\mathscr{H})$ $=\mathscr{F}\left(\mathscr{H}_{+}\right) \otimes \mathscr{F}\left(\mathscr{H}_{-}\right)$. Each $T_{i}$ is symplectic as well as being $\varphi$-symplectic.

If $T_{-}$is of Hilbert-Schmidt class, then $\left(T_{v}\right)_{-}$is of Hilbert-Schmidt class and hence the corresponding Bogolyubov transformation is unitarily implementable by a result of Shale [24, 27]. Furthermore the unitary operator implementing the Bogolyubov transformation commutes with $\Theta\left(\Theta=\Gamma(\varphi)\right.$ being identity on $\mathscr{F}\left(\mathscr{H}_{+}\right)$and $(-1)^{N}$ for a number operator $N$ on $\mathscr{F}\left(\mathscr{H}_{-}\right)$) and hence is $\Theta$-unitary.

Next we assume that $T_{-}$is not of Hilbert-Schmidt class. Since 
$T$ is symplectic, the result of Shale [24] implies that the Fock vacuum state is transformed to a state which yields a representation of the canonical commutation relations disjoint from the original one and hence has no non-zero intertwining operator. Thus there is no bounded invertible $U_{T}$ (be it unitary or $\Theta$-unitary). $\quad$ Q. E. D.

\section{§ 8. Weakly $\Theta$-Unitary Implementability}

Recall that a Bogolyubov transformation $T_{B}=B_{+}+B_{-}$is weakly $\Theta$-unitarily implementable if there exist a complex linear $\Theta$-isometric operator $U_{B}^{-1}$ on $\mathscr{F}(\mathscr{H})$ and a cyclic vector $\Omega_{B} \in \mathscr{F}(\mathscr{H})$ such that

$$
U_{B}^{-1} P\left(\Phi_{\varphi}\left(B f_{1}\right), \cdots, \Phi_{\varphi}\left(B f_{n}\right)\right) \Omega=P\left(\Phi_{\varphi}\left(f_{1}\right), \cdots, \Phi_{\varphi}\left(f_{n}\right)\right) \Omega_{B}
$$

for any polynomial $P$ of (non-commutative) fields and any test functions $f_{j}\left(U_{B}^{-1}\right.$ is not necessarily a bounded operator).

For this implementability, we do not have a complete criterion. A necessary condition and a suffcient condition are given by the following theorems :

Theorem 12. If a Bogolyubov transformation $T_{B}$ is weakly $\Theta$ unitarily implementable, then $B_{-}$is of Hilbert-Schmidt class and the negative real axis and zero is in the resolvent set of $B_{+}^{(\varphi)} B_{+}$.

Theorem 13. Bogolyubov transformations $T_{s}$ and $T_{S^{-1}}$ are weakly $\Theta$-unitarily implementable if the following conditions are all satisfied:

(i ) $S_{-}$is of Hilbert-Schmidt class,

(ii) $S$ is a generalized $\varphi$-scaling:

$$
S=\left(\begin{array}{ll}
h & 0 \\
0 & h^{-1}
\end{array}\right)
$$

on $\mathscr{H}=K \oplus J K$ where $K$ is a real linear subspace of $\mathscr{H}$ such that $K \perp J K$ with resect to $(,)_{r}$ and $\langle,\rangle_{r}$.

(iii) The spectrum of the selfadjoint part $\alpha_{r}=\left(\alpha+\alpha^{*}\right) / 2$ of $\alpha=h^{-2}$ is in $\left[c, c^{-1}\right]$ for some $1 \geqq c>0$.

Remark 4. For the necessity of the positivity of $\alpha_{r}$, see Theorem 
27.

In connection with the discussion of $\Theta$-unitary quasi-implementability, we compute the overlap of the vacuum $\Omega$ and the transformed vector $\Omega_{s}$ as follows :

Theorem 14. Under the condition of Theorem 13, the overlap $\left|\left\langle\Omega, \Omega_{s}\right\rangle\right|$ of the Fock vacuum $\Omega$ and the transformed vacuum $\Omega_{s}$ is given by

$$
\operatorname{det}^{-1 / 2}\left(\frac{h+h^{-1}}{2}\right),
$$

which is non-vanishing finite. Further for $B=V_{1} S V_{2}$ with $V_{i} \varphi$-unitary, if $\Omega_{B}$ exists, then

$$
\begin{gathered}
\left|\left\langle\Omega, \Omega_{B}\right\rangle\right|=\left|\left\langle\Omega, \Omega_{S}\right\rangle\right|=\operatorname{det}^{-1 / 2}\left(\frac{h+h^{-1}}{2}\right) \\
=\operatorname{det}^{-1 / 4}\left(1+B_{-}^{(\varphi)} B_{-}\right)=\operatorname{det}^{-1 / 4}\left(B_{+}^{(\varphi)} B_{+}\right) .
\end{gathered}
$$

Remark 5. When $\varphi=1$, from Theorems 12 and 13 , we see that symplectic operator $T_{B}$ is unitarily implementable if and only if $B_{-}$is in the Hilbert-Schmidt class [Shale]. Further

$$
1 \leqq \operatorname{det}\left(1+B_{-}^{*} B_{-}\right)<\infty
$$

if and only if $B_{-}$is in the Hilbert-Schmidt class, and hence $T_{B}$ is unitarily implementable if and only if the overlap is non-vanishing.

In these theorems $\Omega_{s}$ is well defined by the following lemma:

Lemma 15. Under the assumption of Theorem 13, a vector $\Omega_{s}$ in the Fock space, which is cyclic for the polynomials of fields and satisfies

$$
\begin{aligned}
& \left\langle\Omega_{s}, P\left(\Phi_{\varphi}\left(f_{1}\right), \cdots, \Phi_{\varphi}\left(f_{n}\right)\right) \Omega_{S}\right\rangle \\
& =\left\langle\Omega, P\left(\Phi_{\varphi}\left(S f_{1}\right), \cdots, \Phi_{\varphi}\left(S f_{n}\right)\right) \Omega\right\rangle
\end{aligned}
$$

for all polynomials $P$ and test functions $f_{1}, \cdots, f_{n}$ is unique up to a multiply of identity. 
The following theorem means that the vacuum $\Omega_{s}$ is in the domain of the number operator $N$.

Theorem 16. Under the assumption of Theorem 13,

$$
\Omega_{s} \in D(N)
$$

where $N$ is the number operator.

The proof of Theorem 13 involves the "Q-space" method, which we shall discuss in the next section. The proofs of Lemma 15, which is related to Theorem 13, and Theorems 13, 14 and 16 will be given in $\S 10$. We shall prove Theorem 12 in this section.

Proof of Theorem 12. We shall use the following well known property of the Fock vacuum vector $\Omega$ :

$$
\left[a^{*}(f)\right]^{(\theta)} \Omega=\frac{1}{\sqrt{2}}\left(\Phi_{\varphi}(f)+i \Phi_{\varphi}(J f)\right) \Omega=0
$$

for all $f \in \mathscr{H}$. By definition $\Omega_{B}$ satisfies

$$
\left(\Phi_{\varphi}\left(B^{-1} f\right)+i \Phi_{\varphi}\left(B^{-1} J f\right)\right) \Omega_{B}=0
$$

for all $f \in \mathscr{H}$.

To show that $B_{-}$is of Hilbert-Schmidt class, let

$$
\Omega_{B}=\oplus \omega_{(m)}, \omega_{(m)} \in \mathscr{F}(m)
$$

where $\mathscr{F}^{(n)}=S_{n}\left(\otimes_{n} \mathscr{H}\right)$ is the $n$-particle space. Then (8-5) implies

$$
\begin{gathered}
\left\langle\left(B^{-1}\right)_{+} f, \omega_{(1)}\right\rangle=0, \\
\sqrt{m+1} W_{m+1} \cdot\left(B^{-1}\right)_{+} f+\sqrt{m} S_{m}\left[\left(\left(B^{-1}\right)_{-} f\right) \otimes \omega_{(m-1)}\right]=0
\end{gathered}
$$

where $m \geqq 1$ and $W_{m}$ is the following mapping of $\mathscr{H}$ into $S_{(m-1)}$ $\times\left(\bigotimes_{m-1} \mathscr{H}\right)$ defined by the vector $\omega_{(m)}$ in $S_{m}\left(\bigotimes_{m} \mathscr{H}\right)$ through the characterizing equation

$$
\left\langle S_{m-1}\left(g_{1} \otimes \cdots \otimes g_{m-1}\right), W_{m} g\right\rangle=\left\langle S_{m}\left(g_{1} \otimes \cdots \otimes g_{m-1} \otimes g\right), \omega_{(m)}\right\rangle
$$

relative to the inner product $\langle\rangle=,(, \Theta)$. In particular $W_{2}$ is in the Hilbert-Schmidt class.

First we show that the kernel of $B_{+}$is $\{0\}$. Assume $B_{+} f=0$ for $f \in \mathscr{H}$. Then 


$$
\begin{aligned}
a^{*}(f) \Omega_{B} & =\frac{1}{\sqrt{2}}\left(\Phi_{\varphi}(f)-i \Phi_{\varphi}(J f)\right) \Omega_{B} \\
& =\frac{1}{\sqrt{2}} U_{B}^{-1}\left(\Phi_{\varphi}(B f)-i \Phi_{\varphi}(B J f)\right) \Omega \\
& =\frac{1}{\sqrt{2}} U_{B}^{-1}\left(\Phi_{\varphi}\left(B_{-} f\right)+i \Phi_{\varphi}\left(J B_{-} f\right)\right) \Omega=0
\end{aligned}
$$

due to $B_{+} f=0$ and the equation (8-5) (with $f$ replaced by $B_{-} f$ ). $A$ vector in the Fock space which is annihilated by a creation operator $a^{*}(f)$ must vanish, which contradicts to

$$
\langle\Omega, \Omega\rangle=\left\langle\Omega_{B}, \Omega_{B}\right\rangle=1 \text {. }
$$

The first consequence of this result is that the range of $\left(B^{-1}\right)_{+}$is dense because

$$
\left\langle f,\left(B^{-1}\right)+g\right\rangle=0
$$

for all $g \in \mathscr{H}$ implies

$$
0=\left[\left(B^{-1}\right)_{+}\right]^{(\varphi)} f=B_{+} f
$$

where we have used $B^{-1}=J B^{(\varphi)} J^{-1}=B_{+}^{(\varphi)}-B_{-}^{(\varphi)}$.

As a consequence $\omega_{(m)}=0$ for all odd $m$ : For, (8-6) implies $\omega_{(1)}=0$ by the density of the range of $\left(B^{-1}\right)_{+}$. The relation (8-7) then recursively implies $\omega_{(m)}=0$ for all odd $m$ again due to the density of the range of $\left(B^{-1}\right)_{+}$.

Since $\omega_{0}=0$ would imply $\omega_{(m)}=0$ for all even $m$ by the same recursive argument and this would contradict with (8-8). Hence $\omega_{0} \neq 0$.

We can now use (8-4) for $m=1$ to obtain

$$
\omega_{0}\left(B^{-1}\right)_{-} f=-\sqrt{2 W_{2}} \cdot\left(B^{-1}\right)_{+} f .
$$

Since $W_{2}$ is in the Hilbert-Schmidt class, so is $\left(B^{-1}\right)_{-}$. Hence

$$
B_{-}=\left(J\left(B^{-1}\right)_{-} J^{-1}\right)^{(\varphi)}
$$

is also in the Hilbert-Schmidt class.

Now we assume that $B_{+}^{(\varphi)} B_{+} g=-\lambda g$ with $\lambda \geqq 0, \mid l g \|=1$ and we shall derive a contradiction. First we consider the case $\lambda>0$.

Let $L$ be the set of all vectors satisfying $B_{+}^{(\varphi)} B_{+} g=-\lambda g$. For $g \in L$, we have 


$$
\begin{aligned}
& B_{+}^{(\varphi)} B_{+} B_{+}^{(\varphi)} B_{-} g=B_{+}^{(\varphi)} B_{+} B_{-}^{(\varphi)} B_{+} g \\
& =B_{+}^{(\varphi)} B_{-} B_{+}^{(\varphi)} B_{+} g=-\lambda B_{+}^{(\varphi)} B_{-} g
\end{aligned}
$$

and hence $B_{+}^{(\varphi)} B_{-} g$ again belongs to $L$. Namely $B_{+}^{(\varphi)} B_{-}$is a HilbertSchmidt operator leaving $L$ invariant. Furthermore

$$
\left(B_{+}^{(\varphi)} B_{-}\right)^{2}=B_{+}^{(\varphi)} B_{+}\left(B_{+}^{(\varphi)} B_{+}-1\right) .
$$

Hence

$$
\left\{B_{+}^{(\varphi)} B_{-} \pm[\lambda(\lambda+1)]^{1 / 2}\right\} L
$$

is in the eigen space of $B_{+}^{(\varphi)} B_{-}$belonging to the eigenvalue $\pm[\lambda(\lambda+1)]^{1 / 2}$, and at least one of them is non-zero. Let $g$ be a non-zero vector in one of these spaces. Then $\mathrm{f}_{ \pm} \equiv B_{ \pm}^{(\varphi)} f, f \equiv B_{-}$g has the following property :

$$
\begin{aligned}
& f_{+}= \pm[\lambda(\lambda+1)]^{1 / 2} g, \\
& f_{-}=-(1+\lambda) g .
\end{aligned}
$$

By (8-5), we have

$$
\left[\Phi_{\varphi}\left(B^{-1} f\right)+i \Phi_{\varphi}\left(B^{-1} J f\right)\right] \Omega_{B}=0 .
$$

By the canonical commutation relation

$$
\begin{aligned}
& {\left[\Phi_{\varphi}\left(g_{1}\right), \Phi_{\varphi}\left(g_{2}\right)^{*}\right]=\left[\Phi_{\varphi}\left(g_{1}\right), \Phi_{\varphi}\left(\varphi g_{2}\right)\right]} \\
& =i \operatorname{Im}\left\langle g_{1}, \varphi g_{2}\right\rangle=-i\left(g_{1}, J g_{2}\right)_{r},
\end{aligned}
$$

we obtain

$$
\begin{aligned}
& {\left[\left\{\Phi_{\varphi}\left(B^{-1} f\right)+i \Phi_{\varphi}\left(B^{-1} J f\right)\right\},\left\{\Phi_{\varphi}\left(B^{-1} f\right)+i \Phi_{\varphi}\left(B^{-1} J f\right)\right\}^{*}\right]} \\
& =2\left(B^{-1} J f, J B^{-1} f\right)_{r}=2\left(J B^{(\varphi)} J^{-1} f, B^{(\varphi)} f\right)_{r}=2\left(f_{+}-f_{-}, f_{+}+f_{-}\right)_{r} \\
& =2\left(\left.\left\|f_{+}\right\|\right|^{2}-\left\|f_{-}\right\|^{2}\right)=-2(1+\lambda)\|g\|^{2},
\end{aligned}
$$

where we have used $(h, J h)_{r}=0$ for any $h \in \mathscr{H}$ and $J B^{-1} J^{-1}=B^{(\varphi)}=$ $B_{+}^{(\varphi)}+B_{-}^{(\varphi)}$.

Combining (8-9) and (8-11), we obtain the following contradiction:

$$
0 \leqq\left\|\left.\left\{\Phi_{\varphi}\left(B^{-1} f\right)+i \Phi_{\varphi}\left(B^{-1} J f\right)\right\}^{*} \Omega_{B}\right|^{2}=-2(1+\lambda)\right\| g\left\|\left.\right|^{2}|| \Omega_{B}\right\|^{2}<0 .
$$

This proves that $B_{+}^{(\varphi)} B_{+}$does not have negative eigenvalues.

Finally consider the case $\lambda=0$. By the same computation as the previous case, we obtain 


$$
\left(B_{+}^{(\varphi)} B_{-}\right) g_{1}=0,\left(B_{+}^{(\varphi)} B_{+}\right) g_{1}=0
$$

for $g_{1} \equiv\left(B_{+}^{(\varphi)} B_{-}\right) g$. Because $B_{+}^{(\varphi)} B_{-}=B_{-}^{(\varphi)} B_{+}$, we obtain

$$
\left(B_{+}^{(\varphi)}+B_{+}^{(\varphi)}\right) B_{+} g_{1}=B^{(\varphi)} B_{+} g_{1}=0 .
$$

By the invertibility of $B^{(\varphi)}$, we obtain $B_{+} g_{1}=0$. Since $\operatorname{Ker}\left(B_{+}\right)=\{0\}$, $g_{1}=0$. Thus

$$
B_{+}^{(\varphi)} B_{-} g=0, B_{+}^{(\varphi)} B_{+} g=0 .
$$

The same argument as above now shows that $g=0$. Therefore 0 is not an eigenvalue of $B_{+}^{(\varphi)} B_{+}$. Since $B_{+}^{(\varphi)} B_{+}-1=B_{-}^{(\varphi)} B_{-}$is compact, this shows that the negative axis and zero are in the resolvent set of $B_{+}^{(\varphi)} B_{+}$.

Q. E. D.

\section{$\S$ 9. Q-Space Method}

As a preparation of the full proof of Theorems 13, 14 and 16, we prove them for a special case of finite dimensional $\mathscr{H}$ using the following $Q$-space method.

Corresponding to any direct orthogonal decomposition $\mathscr{H}=K_{0} \oplus J K_{0}$ with respect to the positive definite inner product $(,)_{r}$, we have a maximal abelian algebra generated by (the spectral projections of) selfadjoint fields

$$
\Phi(f)=2^{-1 / 2}\left(a^{*}(f)+\left(a^{*}(f)\right)^{*}\right),
$$

and hence we can identify the Fock space as a certain $L^{2}$ space where fields $\Phi(f)$ with $f \in K_{0}$ are multiplication of certain functions. We shall use such a structure in this section to discuss $\mathscr{F}(\mathscr{H})$ with indefinite metric $\langle\rangle=,(, \Theta)$ and the $\Theta$-selfadjoint fields $\Phi_{\varphi}(f)$.

If we fix an orthonormal basis $e_{1}, \cdots, e_{n}$ in $K_{0}$ relative to the definite metric, there exists a unitary map $W$ from $\mathscr{F}(\mathscr{H})$ onto $L^{2}\left(Q, d \mu_{0}\right)$ such that

$$
W P\left(\Phi\left(e_{1}\right), \Phi\left(e_{2}\right), \cdots, \Phi\left(e_{n}\right)\right) \Omega=P\left(q_{1}, q_{2}, \cdots, q_{n}\right)
$$

for any polynomial $P$ where $Q=R^{n}$ and

$$
d \mu_{0}=\pi^{-n / 2} \exp \left(-\sum q_{i}^{2}\right) \Pi d q_{i} .
$$


Let $\mathscr{H}=K \oplus J K, K$ and $J K$ be $\varphi$-invariant and mutually orthogonal, $K_{ \pm}=P_{ \pm} K$ and $K_{0}=K_{+} \oplus J K_{-} . \quad W$ should implement

$$
W \Phi_{\varphi}\left(e_{i}\right)\left\{\operatorname{resp} . \Phi_{\varphi}\left(J e_{\imath}\right)\right\} W^{-1}=\left\{\begin{array}{r}
q_{i}\left\{\text { resp. }-i \frac{\partial}{\partial q_{i}}+i q_{i}\right\} \text { for } e_{i} \in K_{+}, \\
-i q_{i}\left\{\text { resp. }-\frac{\partial}{\partial q_{\imath}}+q_{i}\right\} \text { for } e_{i} \in K_{-} .
\end{array}\right.
$$

Let $\psi=P_{+}+i P_{-}$. Then $\phi$ commutes with $\varphi$ and $\psi^{*}=\psi^{-1}, \psi^{2}=\varphi$. For the decomposition of $S$ relative to $K \oplus J K, \psi^{*} h^{-1} \psi$ is $\varphi$-selfadjoint and symmetric in the sense that $A^{\text {tr }} \equiv C A^{*} C=A$ for the complex conjugation $C=(+1) \oplus(-1)$ on $K \oplus J K$ and $A=\psi^{*} h^{-1} \psi$.

We introduce a matrix $\alpha$ by $(q, \alpha q)=\sum_{i,} \alpha_{\imath}, q_{i} q_{\text {, where }} \alpha_{\imath \jmath}=$ $\left(e_{\imath}, \psi^{*} h^{-2} \psi e_{j}\right)$ is symmetric due to $\left(\psi^{*} h^{-2} \psi\right)^{t r}=\psi^{*} h^{-2} \psi$ and $C e_{\jmath}=e_{\jmath}$.

We claim that

$$
\Omega_{S}=[\operatorname{det} \alpha]^{1 / 4} \exp \left[-\frac{1}{2}(q,(\alpha-1) q)\right]
$$

has the following two properties:

(i) For $n \geqq 0$, and any polynomial $P$.

$$
P\left(q_{1}, \cdots, q_{n}\right) \Omega_{S} \in L^{2}\left(Q, d \mu_{0}\right),
$$

(ii) For $f \in \mathscr{H}$,

$$
\left(\Phi_{\varphi}\left(S^{-1} f\right)+i \Phi_{\varphi}\left(S^{-1} J f\right)\right) \Omega_{S}=0 .
$$

The property (i) is immediate from the definition due to the assumption that zero and negative real number are not eigenvalues of $\alpha_{r}$ $=\left(\alpha+\alpha^{*}\right) / 2$ and hence $\Omega_{s}$ has Gaussian fall-off for large $|q|$. The property (ii) follows immediately

Proof of Theorem 13 and Lemma 15 for finite dimensional $\mathscr{H}$. We define an operator $U_{s}^{-1}$ by

$$
\begin{aligned}
& U_{S}^{-1} P\left(\Phi_{\varphi}\left(f_{1}\right), \cdots, \Phi_{\varphi}\left(f_{n}\right)\right) \Omega \\
& \quad=P\left(\Phi_{\varphi}\left(S^{-1} f_{1}\right), \cdots, \Phi_{\varphi}\left(S^{-1} f_{n}\right)\right) \Omega_{s},
\end{aligned}
$$

where the polynomial $P$ and test functions $f_{1}, \cdots, f_{n}$ are arbitrary. Since any polynomial annihilating $\Omega$ is known to belong to the left ideal generated by annilhiation operators $\Phi_{\varphi}(f)+i \Phi_{\varphi}(J f)$, (9-5) guarantees that zero vectors are mapped to zero vectors by $U_{s}^{-1}$ and 
hence $U_{s}^{-1}$ is a well-defined linear operator.

Since $\Omega_{s} \neq 0$ for all $q$ and $\Omega_{s} \exp \left[-\frac{1}{2} \sum q_{j}^{2}\right]$ has a Gaussian decrease at $q=\infty, \Omega_{s}$ is cyclic for the polynomial $P$ in $L^{2}\left(Q, d \mu_{0}\right)$. Hence the image of $U_{S}^{-1}$ is dense in $L^{2}\left(Q, d \mu_{0}\right)$.

Next (9-5) and the canonical commutation relations uniquely determine

$$
\left\langle\Omega_{s}, P\left(\Phi_{\varphi}\left(f_{1}\right), \cdots, \Phi_{\varphi}\left(f_{n}\right)\right) \Omega_{s}\right\rangle
$$

because $\left(\Phi_{\varphi}\left(S^{-1} f\right)+i \Phi_{\varphi}\left(S^{-1} J f\right)\right)$ and its $\Theta$-adjoint $\left(\Phi_{\varphi}\left(S^{-1} f\right)-i \Phi_{\varphi}\right.$ $\left(S^{-1} J f\right)$ ) generate the polynomial algebra. Hence it must be equal to

$$
\left\langle\Omega, P\left(\Phi_{\varphi}\left(S f_{1}\right), \cdots, \Phi_{\varphi}\left(\mathrm{S} f_{n}\right)\right) \Omega\right\rangle
$$

which means that $U_{S}^{-1}$ is $\Theta$-isometric.

Finally to prove that $S^{-1}$ is also weakly $\Theta$-unitarily implementable, let

$$
h^{-2}=\alpha=\alpha_{r}+i \alpha_{i}
$$

where $\alpha_{r}$ and $i \alpha_{\imath}$ are selfadjoint and skew-selfadjoint parts of $\alpha$ respectively. For $S^{-1}$, we have

$$
\alpha^{-1}=\left(\alpha_{r}+i \alpha_{i}\right)^{-1}=\left(\alpha_{r}+\alpha_{i} \alpha_{r}^{-1} \alpha_{i}\right)^{-1}-i \alpha_{r}^{-1} \alpha_{i}\left(\alpha_{r}+\alpha_{i} \alpha_{r}^{-1} \alpha_{i}\right)^{-1} .
$$

Since $\left(\alpha^{-1}\right)_{r}=\left(\alpha_{r}+\alpha_{i} \alpha_{r}^{-1} \alpha_{\imath}\right)^{-1}$ is again a positive operator, $S^{-1}$ is also weakly $\Theta$-unitarily implementable.

Q. E. D.

For the purpose of our proof for the infinite dimensional case, we need a few more estimate about $\Omega_{s}$. First we mention the following two lemmas due to Klein $[15,27]$ :

Lemma 17. Let $\left(Q_{i}, d \mu_{i}\right)$ be a sequence of probability measure spaces. Assume $f_{i} \geqq 0, f_{i} \in L^{2}\left(Q_{i}, d \mu_{i}\right)$, and $\left\|f_{i}\right\|_{2}=1$ for all $i$. Let $F_{j}=\Pi_{i=1}^{j} f_{i}$. If $\Pi_{i=1}^{\infty}\left\|f_{i}\right\|_{p}<\infty$ for some $p>2$, then for some $F \in L^{2} \cap L^{p}$,

$$
F_{j} \rightarrow F
$$

in $L^{2}$.

Lemma 18. Let $f_{\lambda}(q)=\sqrt{\lambda} \exp \left[-\frac{1}{2}\left(\lambda^{2}-1\right) q^{2}\right]$, and let $\left\|_{1}\right\|_{p}$ be the 
norm of $f$ with respect to the probability measure $d \mu_{0}=\pi^{-1 / 2} \exp \left(-q^{2}\right)$ dq. Let $1 \geqq c>0$ be given so that $c \leqq \lambda^{2} \leqq c^{-1}$. Then there is a constant $\eta$ for some $p>2\left(\right.$ any $\left.p<2(1-c)^{-1}\right)$ such that

$$
\left\|f_{\lambda}(q)\right\|_{p} \leqq \exp \left(\eta\left(\lambda^{2}-1\right)^{2}\right) \text {. }
$$

These two lemmas imply that a formal vacuum given by

$$
\Omega_{S}=\Pi \sqrt{\lambda_{i}} \exp \left[-\frac{1}{2}\left(\lambda_{i}^{2}-1\right) q_{i}^{2}\right]
$$

is in $L^{2}$ if $\sum\left(\lambda_{i}^{2}-1\right)^{2}<\infty$, and can be directly applied to our case if $\varphi=1$, because $S$ is a selfadjoint scaling operator in this case $\left(\left\{\lambda_{i}^{2}\right\}\right.$ are eigenvalues of $\alpha$ in this case).

In order to prove the following theorem and as a preparation of the next section, we briefly discuss the compact operators and determinant of Hilbert space operators [see, for example, 6, 23, 25, $26]$.

Let $\mathscr{H}$ be a separable Hilbert space and let $\mathscr{B}(\mathscr{H})$ be the $C^{*}$-algebra consisting of the bounded operators on $\mathscr{H}$. For a complete orthonormal basis $\left\{\phi_{i} ; i=1,2, \cdots\right\}$ in $\mathscr{H}$ relative to the inner product $($,$) , the trace of operators is defined by$

$$
\operatorname{Tr}(A)=\sum_{i}\left(\phi_{i}, A \phi_{i}\right)
$$

If $A$ is positive, selfadjoint, then $\operatorname{Tr}(A)$ does not depend on the choice of the basis.

Let $|A|=\left(A^{*} A\right)^{1 / 2}$, and define

$$
\mathscr{C}_{p}=\left\{A \in \mathscr{B}(\mathscr{H}) ; \operatorname{Tr}\left(|A|^{p}\right)<\infty\right\},
$$

and

$$
! \mid A \|_{p}=\left(\operatorname{Tr}\left(|A|^{p}\right)\right)^{1 / p}
$$

Especially $\mathscr{C}_{2}$ is called the Hilbert-Schmidt class and is a two-sided ideal in $\mathscr{B}(\mathscr{H})$.

We summarize several inequalities without proof $[16,25,26]$. If $A \in \mathscr{C}_{p}, B \in \mathscr{C}_{q}$ with $p^{-1}+q^{-1}=1, p, q \geqq 1$, then both $A B$ and $B A$ are in $\mathscr{C}_{1}$ and Hölder inequality holds: 


$$
|\operatorname{Tr}(A B)| \leqq\|A B\|_{1} \leqq \quad\|A\|_{p}|| B \|_{q} .
$$

If $A \in \mathscr{B}(\mathscr{H}), B \in \mathscr{C}_{p}$, then

$$
\|A B\|_{p} \leqq|| A\left|\||| B\|_{p}, \quad\|B A\|_{p} \leqq\|A\|\|\|_{\|_{p}} .\right.
$$

Let $\left\{\lambda_{i}(A) ; i=1,2, \ldots\right\}$ be a listing of all the non-zero eigenvalues of $A$ counted up to (algebraic) multiplicity. We define

$$
\operatorname{det}(1+A)=\Pi\left(1+\lambda_{i}(A)\right) .
$$

Let $\left\{\mu_{i}(A) ; i=1,2, \ldots\right\}$ be a listing of all the non-zero eigenvalues of $|A|$ counted up to multiplicity. Then

$$
1 \leqq \operatorname{det}(1+|A|)=\Pi\left(1+\mu_{i}(A)\right) \leqq \exp \|A\|_{1},
$$

and $\operatorname{det}(1+|A|)<\infty$ if and only if $A \in \mathscr{C}_{1}$. The following inequality is due to Weyl $[6,26]$ :

$$
\sum\left|\lambda_{i}(A)\right|^{p} \leqq \sum_{i} \mu_{i}(A)^{p}=\|A\|_{p}^{p}
$$

for $p \geqq 1$. Therefore $\operatorname{det}(1+A)$ exists for $A \in \mathscr{C}_{1}$. Further $[6,26]$

$$
|\operatorname{det}(1+A)| \leqq \operatorname{det}\left(1+\mid A_{\mid}\right) \text {. }
$$

Our final theorem in this section is the following that is a generalization of Lemmas 17 and 18 :

Theorem 19. Let $\alpha$ and $\beta$ be bounded symmetric operators (of arbitrarily large but finite rank $n$ ) such that

(i) $0<c \leqq \alpha_{r}, \beta_{r} \leqq c^{-1}, 1 \geqq c>0$,

(ii) $-m \leqq \alpha_{\imath}, \beta_{i} \leqq m$,

(iii) $\|\alpha-1\|_{2},\|\beta-1\|_{2} \leqq \kappa$, for some $\kappa<\infty$ independent of $n$. Here $\alpha_{r}$ and $i \alpha_{i}\left(\right.$ resp. $\beta_{r}$ and $i \beta_{i}$ ) are selfadjoint and skew-selfadjoint parts of $\alpha($ resp. $\beta)$ respectively. Let

$$
\begin{aligned}
& f_{\alpha}=(\operatorname{det} \alpha)^{1 / 4} \exp \left[-\frac{1}{2}(q,(\alpha-1) q)\right], \\
& f_{\beta}=(\operatorname{det} \beta)^{1 / 4} \exp \left[-\frac{1}{2}(q,(\beta-1) q)\right],
\end{aligned}
$$

and let $\|\cdots\|_{p}$ be the norm with respect to a probability measure $d \mu_{0}$ $=\Pi_{i=1}^{\infty} \pi^{-1 / 2} \exp \left[-q_{i}^{2}\right] d q_{i} . \quad I f_{\|} \mid \alpha-\beta \|_{2}$ is sufficiently small, then there is a constant $\eta$ such that 


$$
\left\|f_{\alpha}-f_{\beta}\right\|_{2}^{2} \leqq \eta\|\alpha-\beta\|_{2}^{2}
$$

for an appropriate choice of the quadratic root of $\operatorname{det}(\alpha)$ and $\operatorname{det}(\beta)$.

For the proof of this theorem, following lemmas are useful:

Lemma 20. Under the same assumption of Theorem 19,

$$
\left\|f_{\alpha}\right\|_{p}^{p}=\frac{|\operatorname{det} \alpha|^{p / 4}}{\left[\operatorname{det}\left(1+\frac{p}{2}\left(\alpha_{r}-1\right)\right)\right]^{1 / 2}}
$$

for $p<2(1-c)^{-1}$. Further in this case, there is a finite constant $\eta^{\prime}$ such that

$$
\left\|f_{\alpha}\right\|_{p} \leqq \exp \left[\frac{1}{8 c^{2}}\left\|\alpha_{i}\right\|_{2}^{2}+\eta^{\prime}|| \alpha_{r}-1 \|_{2}^{2}\right] .
$$

Lemma 21. Let $\alpha_{r}$ and $\beta_{r}$ be the operators defined in Theorem 19. Then

$$
\begin{gathered}
\left\|\alpha_{r}^{1 / 2}-\beta_{r}^{1 / 2}\right\|_{2} \leqq \frac{1}{2} c^{-1 / 2}\left\|\alpha_{r}-\beta_{r}\right\|_{29} \\
\left\|\alpha_{r}^{1 / 2}-\left(\frac{\alpha_{r}+\beta_{r}}{2}\right)^{1 / 2}\right\|_{2} \leqq \frac{1}{4} c^{-1 / 2}\left\|\alpha_{r}-\beta_{r}\right\|_{2} \\
\left\|\beta_{r}^{1 / 2}-\left(\frac{\alpha_{r}+\beta_{r}}{2}\right)^{1 / 2}\right\|_{2} \leqq \frac{1}{4} c^{-1 / 2}\left\|\alpha_{r}-\beta_{r}\right\|_{2} .
\end{gathered}
$$

Lemma 22. Let $\alpha_{r}$ and $\beta_{r}$ be as above. Then

$$
1 \leqq K \equiv \frac{\operatorname{det}^{1 / 2}\left(\frac{\alpha_{r}+\beta_{r}}{2}\right)}{\operatorname{det}^{1 / 4}\left(\alpha_{r}\right) \operatorname{det}^{1 / 4}\left(\beta_{r}\right)} \leqq \exp \left[(2 c)^{-4}|| \alpha_{r}-\beta_{r} \|_{2}^{2}\right]
$$

Lemma 23. Let $\alpha=\alpha_{r}+i \alpha_{i}, \beta=\beta_{r}+i \beta_{i}$ be as in Theorem 19. Let

$$
A=\alpha_{r}^{-1 / 2} \alpha_{r} \alpha_{r}^{-1 / 2}, B=\beta_{r}^{-1 / 2} \beta_{i} \beta_{r}^{-1 / 2},
$$

and let

$$
M=\left[\operatorname{det}^{1 / 8}\left(1+A^{2}\right)-\operatorname{det}^{1 / 8}\left(1+B^{2}\right)\right]^{2} .
$$

Then there is a constant $c_{0}$ for sufficiently small $\|\alpha-\beta\|_{2}$ such that 


$$
M \leqq c_{0}\|\alpha-\beta\|_{2}^{2}
$$

(For example, $c_{0}=\exp \left(\frac{\kappa^{2}}{4 c^{2}}\right) \gamma^{2} e^{2 a \gamma}, \gamma=2 m c^{-1}\left(m c^{-2}+c^{-1}\right)$, for $\left.\|\alpha-\beta\|_{2} \leqq a.\right)$

Proof of Lemma 20. Since

$$
\left|f_{\alpha}\right|=|\operatorname{det} \alpha|^{1 / 4} \exp \left[-\frac{1}{2}\left(q,\left(\alpha_{r}-1\right) q\right)\right]
$$

the first equation is obvious, while

$$
\begin{aligned}
|\operatorname{det} \alpha|^{2} & =\operatorname{det}\left(\alpha_{r}+i \alpha_{i}\right) \operatorname{det}\left(\alpha_{r}-i \alpha_{i}\right) \\
& =\operatorname{det}\left(\alpha_{r}^{2}\right) \operatorname{det}\left(1+\alpha_{r}^{-1 / 2} \alpha_{i} \alpha_{r}^{-1} \alpha_{i} \alpha_{r}^{-1 / 2}\right) \\
& \leqq \exp \left[\|\left.\alpha_{r}^{-1 / 2} \alpha_{i} \alpha_{r}^{-1} \alpha_{i} \alpha_{r}^{-1 / 2}\right|_{1}\right] \operatorname{det}\left(\alpha_{r}^{2}\right) \\
& \leqq \exp \left[|| \alpha_{r}^{-2}|||| \alpha_{i}^{2} \|_{1}\right] \operatorname{det}\left(\alpha_{r}^{2}\right)=\exp \left[c^{-2}\left\|\alpha_{i}\right\|_{2}^{2}\right] \operatorname{det}\left(\alpha_{r}^{2}\right)
\end{aligned}
$$

Then

$$
\left\|f_{\alpha}\right\|_{p} \leqq \exp \left[\frac{1}{8 c^{2}}\left\|\alpha_{i}\right\|_{2}^{2}\right]\left[\frac{\left(\operatorname{det}\left(\alpha_{r}\right)\right)^{p / 4}}{\left(\operatorname{det}\left(1+\frac{p}{2}\left(\alpha_{r}-1\right)\right)^{1 / 2}\right.}\right]^{1 / p}
$$

Hence we obtain the desired inequality:

$$
\left\|f_{\alpha}\right\|_{p} \leqq \exp \left[\frac{1}{8 c^{2}}\left\|\alpha_{\imath}\right\|_{2}^{2}+\eta^{\prime}\left\|\left(\alpha_{r}-1\right)\right\|_{2}^{2}\right]
$$

Q. E. D.

Proof of Lemma 21. Let $\left\{\phi_{i}\right\}$ be the complete orthonormal eigenvectors of $\alpha_{r}^{1 / 2}+\beta_{r}^{1 / 2}$, and let $\left\{\lambda_{i}\right\}$ be the eigenvalues. Then $2 c^{1 / 2} \leqq \lambda_{i}$ $\leqq 2 c^{-1 / 2}$. Since

$$
\alpha_{r}-\beta_{r}=\frac{1}{2}\left[\left(\alpha_{r}^{1 / 2}+\beta_{r}^{1 / 2}\right)\left(\alpha_{r}^{1 / 2}-\beta_{r}^{1 / 2}\right)+\left(\alpha_{r}^{1 / 2}-\beta_{r}^{1 / 2}\right)\left(\alpha_{r}^{1 / 2}+\beta_{r}^{1 / 2}\right)\right],
$$

we have

$$
\begin{aligned}
& || \alpha_{r}-\beta_{r} \|_{2}^{2}=\sum_{i, j}\left|\left(\phi_{i}, \quad\left(\alpha_{r}-\beta_{r}\right) \phi_{j}\right)^{2}\right| \\
& =\sum_{i, j}\left(\frac{\lambda_{i}+\lambda_{j}}{2}\right)^{2}\left|\left(\phi_{i}, \quad\left(\alpha_{r}^{1 / 2}-\beta_{r}^{1 / 2}\right) \phi_{j}\right)^{2}\right| \\
& \geqq 4 c \sum_{i, j}\left|\left(\phi_{i}, \quad\left(\alpha_{r}^{1 / 2}-\beta_{r}^{1 / 2}\right) \phi_{j}\right)^{2}\right|=4 c|| \alpha_{r}^{1 / 2}-\beta_{r}^{1 / 2} \|_{2}^{2} .
\end{aligned}
$$

Other inequalities follow by substitution.

Q. E. D. 
Proof of Lemma 22. Let $x=\alpha_{r}^{-1 / 2} \beta_{r} \alpha_{r}^{-1 / 2}>0$. Then $\left\|x^{-1}\right\| \leqq c^{-2}$, and

$$
\begin{aligned}
& 1 \leqq K=\operatorname{det}^{1 / 4}\left(1+\frac{1}{4} x^{-1}(x-1)^{2}\right) \leqq \exp \left[\frac { 1 } { 1 6 } \left\|x^{-1}\left|\left\|\mid(x-1)^{2}\right\|_{1}\right]\right.\right. \\
& \leqq \exp \left[\frac{1}{16}\left\|x^{-1}\right\|\left\|\alpha_{r}^{-1 / 2}\left(\alpha_{r}-\beta_{r}\right) \alpha_{r}^{-1 / 2}\right\|_{2}^{2}\right] \\
& \leqq \exp \left[\frac{1}{16}\left|i x^{-1}\right| i|| \alpha_{r}^{-2}\|\| \alpha_{r}-\beta_{r} \|_{2}^{2}\right] \leqq \exp \left[(2 c)^{-4}\left\|\alpha_{r}-\beta_{r}\right\|_{2}^{2}\right] .
\end{aligned}
$$

Q. E. D.

\section{Proof of Lemma 23.}

$$
\begin{aligned}
& M=\operatorname{det}^{1 / 4}\left(1+A^{2}\right)\left\{\operatorname{det}\left[1-\left(1+A^{2}\right)^{-1 / 2}\left(A^{2}-B^{2}\right)\left(1+A^{2}\right)^{-1 / 2}\right]-1\right\}^{2} \\
& \leqq \exp \left[\frac{1}{4}\|A\|_{2}^{2}\right]\left[\exp || A^{2}-B^{2} \|_{1}-1\right]^{2} .
\end{aligned}
$$

Note $A, B \in \mathscr{C}_{2}$. By Hölder inequality,

$$
\left\|A^{2}-B^{2}\right\|_{1} \leqq\|A+B\|_{2}\|A-B\|_{2} \leqq 2 m c^{-1}\|A-B\|_{2}
$$

Further

$$
\begin{aligned}
\mid: A-B \|_{2} & =\| \alpha_{r}^{-1 / 2} \alpha_{i} \alpha_{r}^{-1 / 2}-\left.\beta_{r}^{-1 / 2} \beta_{i} \beta_{r}^{-1 / 2}\right|_{2} \\
& =\| \alpha_{r}^{-1 / 2}\left(-\alpha_{r}^{1 / 2}+\beta_{r}^{1 / 2}\right) \beta_{r}^{-1 / 2} \alpha_{i} \alpha_{r}^{-1 / 2}+\beta_{r}^{-1 / 2}\left(\alpha_{i}-\beta_{i}\right) \alpha_{r}^{-1 / 2} \\
& \quad+\beta_{r}^{-1 / 2} \beta_{i} \alpha_{r}^{-1 / 2}\left(-\alpha_{r}^{1 / 2}+\beta_{r}^{1 / 2}\right) \beta_{r}^{-1 / 2} \|_{2} \\
& \leqq\left(\left\|\alpha_{r}^{-1 / 2}\right\|_{i}^{\prime} \mid \beta_{r}^{-1 / 2} \alpha_{i} \alpha_{r}^{-1 / 2}\|+\| \beta_{r}^{-1 / 2}\|\| \beta_{r}^{-1 / 2} \beta_{i} \alpha_{r}^{-1 / 2} \|\right) \\
& \times\left\|\alpha_{r}^{1 / 2}-\beta_{r}^{1 / 2}\right\|_{2}+\left\|\alpha_{r}^{-1 / 2}\right\|\left\|\beta_{r}^{-1 / 2}\right\|\left\|\alpha_{i}-\beta_{i}\right\|_{2} \\
& \leqq 2 m c^{-3 / 2}\left\|\alpha_{r}^{1 / 2}-\beta_{r}^{1 / 2}\right\|_{2}+c^{-1}\left\|\alpha_{i}-\beta_{i}\right\|_{2}
\end{aligned}
$$

Then by Lemma 21, we finally have

$$
\|A-B\|_{2} \leqq m c^{-2}|| \alpha_{r}-\beta_{r}\left\|_{2}+c^{-1}\right\| \alpha_{i}-\beta_{i}\left\|_{2} \leqq\left(m c^{-2}+c^{-1}\right)\right\| \alpha-\beta \|_{2} .
$$

Thus

$$
\left\{\exp _{\|}|| A^{2}-\left.B^{2}\right|_{\left.\right|_{2}}-1\right\}^{2} \leqq\left\{\exp \left[2 m c^{-1}\left(m c^{-2}+c^{-1}\right)\|\alpha-\beta\|_{2}\right]-1\right\}^{2},
$$

and then there is a suitable constant $d$ such that

$$
\leqq d|| \alpha-\left.\beta\right|_{2} ^{2}
$$

We set $c_{0}=d \exp \left[\left.\frac{1}{4}|| A\right|_{1^{2}} ^{\prime 2}\right]$ to complete the proof. Q. E. D.

Proof of Theorem 19. 


$$
\begin{aligned}
& \left\|f_{\alpha}-f_{\beta}\right\|_{2}^{2}=\left\|f_{\alpha}\right\|_{2}^{2}+\left\|f_{\beta}\right\|_{2}^{2}-\int \bar{f}_{\alpha} f_{\beta} d \mu_{0}-\int f_{\alpha} \bar{f}_{\beta} d \mu_{0} \\
& =\left\{\left|\frac{\operatorname{det}(\alpha)}{\operatorname{det}\left(\alpha_{r}\right)}\right|^{1 / 4}-\left|\frac{\operatorname{det}(\beta)}{\operatorname{det}\left(\beta_{r}\right)}\right|^{1 / 4}\right\}^{2} \\
& +2\left|\operatorname{det}(\alpha) \operatorname{det}(\beta) \operatorname{det}^{-1}\left(\alpha_{r}\right) \operatorname{det}^{-1}\left(\beta_{r}\right)\right|^{1 / 4} \\
& -\left\{\operatorname{det}^{1 / 4}(\bar{\alpha}) \operatorname{det}^{1 / 4}(\beta) \operatorname{det}^{-1 / 2}\left(\frac{\bar{\alpha}+\beta}{2}\right)+(\bar{\alpha} \rightarrow \alpha, \beta \rightarrow \beta)\right\}
\end{aligned}
$$

Let

$$
\mathrm{A}=\alpha_{r}^{-1 / 2} \alpha_{\imath} \alpha_{r}^{-1 / 2}, B=\beta_{r}^{-1 / 2} \beta_{i} \beta_{r}^{-1 / 2}, C=\left(\frac{\alpha_{r}+\beta_{r}}{2}\right)^{-1 / 2}\left(\frac{\alpha_{i}-\beta_{i}}{2}\right)\left(\frac{\alpha_{r}+\beta_{r}}{2}\right)^{-1 / 2} .
$$

Then these are in $\mathscr{C}_{2}$, and

$$
\|C\|_{2}^{2} \leqq \frac{1}{4} c^{-2}|| \alpha_{i}-\beta_{i} \|_{2}^{2}
$$

We have

$$
\begin{aligned}
& \left\|f_{\alpha}-f_{\beta}\right\|_{2}^{2}=\left\{\operatorname{det}^{1 / 8}\left(1+A^{2}\right)-\operatorname{det}^{1 / 8}\left(1+B^{2}\right)\right\}^{2}+2 \operatorname{det}^{1 / 8}\left(1+A^{2}\right) \operatorname{det}^{1 / 8}\left(1+B^{2}\right) \\
- & \frac{\operatorname{det}^{1 / 4}\left(\alpha_{r}\right) \operatorname{det}^{1 / 4}\left(\beta_{r}\right)}{\operatorname{det}^{1 / 2}\left(\frac{\alpha_{r}+\beta_{r}}{2}\right)} \operatorname{det}^{-1 / 2}\left(1+C^{2}\right) \\
\times & {\left[\operatorname{det}^{1 / 4}(1-i A) \operatorname{det}^{1 / 4}(1+i B) \operatorname{det}^{1 / 2}(1+i C)+(A, B, C \rightarrow-A,-B,-C)\right] . }
\end{aligned}
$$

Let

$$
l=\operatorname{det}^{1 / 4}(1-i A) \operatorname{det}^{1 / 4}(1+i B) \operatorname{det}^{1 / 2}(1+i C)
$$

Then

$$
|l|=\operatorname{det}^{1 / 8}\left(1+A^{2}\right) \operatorname{det}^{1 / 8}\left(1+B^{2}\right) \operatorname{det}^{1 / 4}\left(1+C^{2}\right)
$$

and $l$ is given as

$$
|l| \omega,|\omega|=1 .
$$

Let $\omega_{r}=\operatorname{Re} \omega$. Then we finally have

$$
|| f_{\alpha}-f_{\beta} \|_{2}^{2}=M+2|l| \operatorname{det}^{-1 / 2}\left(1+C^{2}\right) K^{-1}\left[\operatorname{det}^{1 / 4}\left(1+C^{2}\right) K-\omega_{r}\right],
$$

where $M$ and $K$ are quantities given in Lemmas 22 and 23 . By previous lemmas, it suffices to prove that, if $|\alpha \alpha-\beta|_{12}^{12}$ is sufficiently small,

$$
\left|\omega_{r}-1\right| \leqq \eta_{1}|| \alpha-\left.\beta\right|_{2} ^{2}
$$


for some constant $\eta_{1}$.

For this purpose, note that

$$
\begin{aligned}
l & =\operatorname{det}^{1 / 4}(1+i C)(1-i A)(1+i B)(1+i C) \\
& =\operatorname{det}^{1 / 4}\left(1+A^{2}+H\right)
\end{aligned}
$$

where

$$
\begin{aligned}
H & =A(B-A)-i(A-B-2 C)+i C A B+i A B C+(A-B) C+C(A-B) \\
& -C^{2}+i C(A-B) C-C A B C .
\end{aligned}
$$

We prove $H \in \mathscr{C}_{1}$ and $\|! H\|_{1} \leqq \eta_{2}\left\|\alpha-\beta_{\|}\right\|_{2}$ for some constant $\eta_{2}$. Since $A, B$ and $C$ are in $\mathscr{C}_{2}$,

$$
\begin{aligned}
& \|A(A-B)\|_{1} \leqq\|A\|_{2}\|A-B\|_{2} \leqq \text { const. }\|\alpha-\beta\|_{2}, \\
& \|A B C\|_{1} \leqq\|A B\|_{2}\|C\|_{2} \leqq \text { const. }\|\alpha-\beta\|_{2} .
\end{aligned}
$$

Thus it suffices to prove $\|A-B-2 C\|_{1} \leqq \eta_{3}|| \alpha-\beta \|_{2}$ for some constant

$$
\begin{aligned}
\eta_{3}: & A-B-2 C \\
= & \alpha_{r}^{-1 / 2} \alpha_{i} \alpha_{r}^{-1 / 2}-\beta_{r}^{-1 / 2} \beta_{\imath} \beta_{r}^{-1 / 2}-2\left(\frac{\alpha_{r}+\beta_{r}}{2}\right)^{-1 / 2}\left(\frac{\alpha_{i}-\beta_{r}}{2}\right)\left(\frac{\alpha_{r}+\beta_{r}}{2}\right)^{-1 / 2} \\
= & \alpha_{r}^{-1 / 2}\left(\beta_{r}^{1 / 2}-\alpha_{r}^{1 / 2}\right) \beta_{r}^{-1 / 2} \alpha_{i} \alpha_{r}^{-1 / 2}+\beta_{r}^{-1 / 2} \beta_{r} \alpha_{r}^{-1 / 2}\left(\beta_{r}^{1 / 2}-\alpha_{r}^{1 / 2}\right) \beta_{r}^{-1 / 2} \\
+ & \beta_{r}^{-1 / 2}\left(\left(\frac{\alpha_{r}+\beta_{r}}{2}\right)^{1 / 2}-\beta_{r}^{1 / 2}\right)\left(\frac{\alpha_{r}+\beta_{r}}{2}\right)^{-1 / 2}\left(\alpha_{i}-\beta_{i}\right) \alpha_{r}^{-1 / 2} \\
+ & \left(\frac{\alpha_{r}+\beta_{r}}{2}\right)^{-1 / 2}\left(\alpha_{i}-\beta_{i}\right) \alpha_{r}^{-1 / 2}\left(\left(\frac{\alpha_{r}+\beta_{r}}{2}\right)^{1 / 2}-\alpha_{r}^{1 / 2}\right)\left(\frac{\alpha_{r}+\beta_{r}}{2}\right)^{-1 / 2} .
\end{aligned}
$$

Here $\alpha_{i}, \beta_{\imath} \in \mathscr{C}_{2}$, and by Lemma 21, $\left(\frac{\alpha_{r}+\beta_{r}}{2}\right)^{1 / 2}-\alpha_{r}^{1 / 2}$ and $\left(\frac{\alpha_{r}+\beta_{r}}{2}\right)$ $-\beta_{r}^{1 / 2}$ are of Hilbert-Schmidt class whose Hilbert-Schmidt norms are dominated by const. $\|\alpha-\beta\|_{2}$. Then there is a suitable constant $\eta_{3}$ such that $\|A-B-2 C\|_{2} \leqq \eta_{3}\|\alpha-\beta\|_{2}$.

Let $\mathrm{H}_{r}$ and $i H_{i}$ be the selfadjoint and skew-selfadjoint parts of $H$ respectively, and let

$$
\operatorname{det}\left(1+A^{2}+H\right)=\operatorname{det}\left(1+A^{2}+H_{r}\right) \operatorname{det}\left(1+i H_{r}^{\prime}\right),
$$

where

$$
H_{i}^{\prime}=\left(1+A^{2}+H_{r}\right)^{-1 / 2} H_{i}\left(1+A^{2}+H_{r}\right)^{-1 / 2}
$$

is a selfadjoint operator such that

$$
\text { ! }\left|H_{i}^{\prime}\left\|_{1} \leqq\right\|\left(1+A^{2}+H_{r}\right)^{-1}\|\| H\left\|_{1} \leqq \eta_{2}\right\|\left(1+A^{2}+H_{r}\right)^{-1}\right|\||| \alpha-\beta\|_{2} .
$$


Then

$$
\operatorname{det}\left(1+i H_{i}^{\prime}\right)=\operatorname{det}^{1 / 2}\left(1+H_{i}^{\prime 2}\right) \exp \left\{i \operatorname{Tr}\left[\operatorname{Sin}^{-1} H_{i}^{\prime}\left(1+H_{i}^{\prime 2}\right)^{-1 / 2}\right]\right\},
$$

and

$$
\omega_{r}=\cos \frac{1}{4}\left\{\operatorname{Tr}\left[\operatorname{Sin}^{-1} H_{i}^{\prime}\left(1+H_{i}^{\prime 2}\right)^{-1 / 2}\right]\right\},
$$

which completes the proof.

Q. E. D.

\section{$\S 10$. Proof of Main Theorems in $\$ 9$.}

For a generalized $\varphi$-scaling $S$ given by $(6-7), S_{-}$is given by

$$
\frac{1}{2}\left(\begin{array}{lr}
h-h^{-1} & 0 \\
0 & h^{-1}-h
\end{array}\right)
$$

on $K \oplus J K$. Then

(10-2) $\quad S_{-}$is H. S. $\leftrightarrow h-h^{-1}$ is H. S. $\leftrightarrow \alpha-1=h^{-2}-1, \quad \alpha^{-1}-1$ are H. S. $\rightarrow \alpha_{r}-1, \alpha_{i},\left(\alpha^{-1}\right)_{r}-1,\left(\alpha^{-1}\right)_{i}$ are H. S.

Let $E_{n}$ be an increasing sequence of finite dimensional orthogonal projections commuting with $\varphi$ and tending to 1 as $n \rightarrow \infty$. Let $h_{n}=$ $\left(E_{n} \alpha E_{n}\right)^{-1 / 2}+\left(1-E_{n}\right)$ and $S_{n}=h_{n} \oplus h_{n}^{-1}$ on $K \oplus J K$. Then $\left\{S_{n}\right\}$ is a sequence of generalized $\varphi$-scalings of finite rank with $\alpha_{n}=h_{n}^{-2}$ satisfying $\left\|\alpha_{n}-1\right\|_{2}<\kappa, c \leqq\left(\alpha_{n}\right)_{r} \leqq c^{-1}$ for all $n$ and

$$
\left\|\alpha_{m}-\alpha_{n}\right\|_{2} \rightarrow 0
$$

as $m, n \rightarrow \infty$. The sequence of transformed vacua $\left\{\Omega_{S_{n}}\right\}$ with

$$
\Omega_{S_{n}}=\left[\operatorname{det}\left(\alpha_{n}\right)\right]^{1 / 4} \exp \left[-\frac{1}{2}\left(q,\left(\alpha_{n}-1\right) q\right)\right]
$$

is Cauchy is $L^{2}\left(Q, d \mu_{0}\right)$ by Theorem 19 :

$$
\Omega_{s_{n}} \rightarrow \Omega_{s} \in L^{2}\left(Q, d \mu_{0}\right) .
$$

The phase of $\left(\operatorname{det}\left(\alpha_{n}\right)\right)^{1 / 4}$ is chosen so that $\left(\operatorname{det}\left(\alpha_{n}\right)\right)^{1 / 4}>0$ if det $\left(\alpha_{n}\right)>0$. Thus we can prove Theorem 13 and Lemma 15 through Lemmas 24-26:

Lemma 24. Let $P$ be a polynomial of the fields $\left\{\Phi_{\varphi}\left(f_{i}\right)\right\}$. Then 


$$
\operatorname{Sup}_{n}\left\|P \Omega_{S_{n}}\right\|_{2}<\infty .
$$

Proof. It suffices to prove the lemma for a polynomial of $\left\{q_{i}\right\}$. In fact derivative terms $\left\{\partial / \partial q_{i}\right\}$ in $\Phi_{\varphi}\left(f_{i}\right)$ are only to induce linear terms of $\left\{q_{i}\right\}$ as $\sum_{j}\left(\alpha_{n}\right)_{i} q_{j}$ in $P$, which stay in a bounded set of $L^{p}\left(Q, d \mu_{0}\right)$ with $\mathrm{p} \geqq 2: \sum_{j}\left|\left(\alpha_{n}\right)_{i j}\right|^{2} \leqq(1+\kappa)^{2}$ for any $n$ and $i$.

By Hölder inequality

$$
\begin{aligned}
\left\|P \Omega_{S_{n}}\right\|_{2}^{2} & =\int\left|P \Omega_{S_{n}}\right|^{2} d \mu_{0} \\
& \leqq\|P\|_{2 q^{\prime}}^{2}\left\|\Omega_{S_{n}}\right\|_{2 p^{\prime}}^{2}
\end{aligned}
$$

where $p^{\prime}, q^{\prime} \geqq 1$ and $\left(p^{\prime}\right)^{-1}+\left(q^{\prime}\right)^{-1}=1$. By Lemma 20 , there is a constant $p$ in $\left(2,2(1-c)^{-1}\right)$ such that

$$
\left\|\Omega_{S_{n}}\right\|_{p} \leqq \exp \left[\frac{1}{8 c^{2}}\left\|\left(\alpha_{n}\right)_{i}\right\|_{2}^{2}+\eta\left\|_{1}\left(\alpha_{n}\right)_{r}-1\right\|_{2}^{2}\right] .
$$

We put $p^{\prime}=p / 2>1$, which completes the proof.

Q. E. D.

\section{Lemma 25.}

$$
\left\langle\Omega_{s}, P \Omega_{s}\right\rangle=\left\langle\Omega, \pi_{s}(P) \Omega\right\rangle,
$$

where $P=P\left(\Phi_{\varphi}\left(f_{1}\right), \cdots, \Phi_{\varphi}\left(f_{n}\right)\right)$ and $\pi_{s}(P)=P\left(\Phi_{\varphi}\left(S f_{1}\right), \cdots, \Phi_{\varphi}\left(S f_{n}\right)\right)$.

Proof. Let $\Psi_{n}=P \Omega_{S_{n}}$. By the above lemma, $\Psi_{n}$ has a sequence converging to a vector $\Psi$. Since $P$ is a closed operator,

$$
\left\{\Omega_{s}, \Psi\right\} \in \operatorname{graph} \bar{P} .
$$

Then $\Psi$ does not depend on the subsequence and $\Psi_{n}$ weakly converges to

$$
\Psi=P \Omega_{s}, \Omega_{s} \in D(P)
$$

For finite rank $S_{n}$,

$$
\left\langle\Omega_{s_{n}}, P \Omega_{s_{n}}\right\rangle=\left\langle\Omega, \pi_{s_{n}}(P) \Omega\right\rangle .
$$

Then

$$
\left\langle\Omega_{s}, P \Omega_{s}\right\rangle=\left\langle\Omega, \pi_{s}(P) \Omega\right\rangle .
$$


Q. E. D.

Lemma 26. $\Omega_{s}$ is a cyclic vector for $\mathscr{F}$.

Proof. First note that $\left\{\overline{Q \Omega_{s}}\right\}=\left\{\overline{P \Omega_{s}}\right\}$, where $\left\{Q \Omega_{s}\right\}$ denotes the set of $Q(q) \Omega_{s}$ with $Q(q)$ polynomials of $\{q\}$. As we have already proved,

$$
\left\{\overline{Q \Omega_{s}}\right\} \ni \Omega_{s_{0}}
$$

if $\alpha-\alpha_{0}$ is of finite rank and $c \leqq(\alpha)_{r},\left(\alpha_{0}\right)_{r} \leqq c^{-1}$ for some $1 \geqq c>0$. Let $E_{n}$ be an increasing sequence of finite dimensional orthogonal projections tending to 1 as $n \rightarrow \infty$ and commuting with $\varphi$. Let $\alpha_{n}=$ $E_{n}+\left(1-E_{n}\right) \alpha\left(1-E_{n}\right)$. Let $h_{n}=\left(\alpha_{n}\right)^{-1 / 2}$ and $S_{n}=h_{n} \oplus h_{n}^{-1}$ on $K \oplus J K$. Therefore $\left\{\overline{Q \Omega_{s}}\right\} \ni \Omega_{S_{n}}$ and $\left\|\alpha_{n}-1\right\|_{2} \rightarrow 0$ as $n \rightarrow \infty$, which implies $\Omega_{s_{n}} \rightarrow \Omega$ in $L^{2}\left(Q, d \mu_{0}\right)$ and

$$
\left\{\overline{Q \Omega_{s}}\right\} \ni \Omega
$$

By the explicit evaluation of $\left\|q^{n} Q \Omega_{s}\right\|_{2}$ as a function of $n$, it is seen that $Q \Omega_{S}$ for any polynomial $Q$ of $\{q\}$ is an (entire) analytic vector of $q_{j^{\circ}}$ Hence $\exp \left(i a q_{j}\right) Q \Omega_{s} \in\left\{\overline{Q \Omega_{s}}\right\}$ for any real $a$. This shows that $\left\{\overline{Q \Omega_{S}}\right\}$ is invariant under the commutative von Neumann algebra $M$ generated by $\left\{\exp \left(i a q_{j}\right)\right\}$. Since the Fock vacuum is known to be cyclic for $M,\left\{\overline{Q \Omega_{s}}\right\} \ni \Omega$ implies that $\left\{\overline{Q \Omega_{S}}\right\}$ is the whole Fock space $\mathscr{F}$.

Q. E. D.

To complete the proof of Theorem 12, it suffices to prove that $T_{s^{-1}}$ is again weakly $\Theta$-unitarily implementable. This is obvious since if $\alpha-1$ is H.S., then $\alpha^{-1}-1$ is again H.S. (see (10-2)) and if $\alpha_{r}$ is strictly positive, then $\left(\alpha^{-1}\right)_{r}$ is again strictly positive as we have already proved in finite dimensional case. $\quad$ Q. E. D.

Proof of Theorem 14.

For finite rank $S_{n}$,

$$
\left\langle\Omega, \Omega_{S_{n}}\right\rangle=\operatorname{det}^{1 / 4}\left(\alpha_{n}\right) \int \exp \left[-\frac{1}{2}\left(q,\left(\alpha_{n}+1\right) q\right] \Pi \frac{d q_{2}}{\sqrt{\pi}}\right.
$$




$$
\begin{aligned}
& =\operatorname{det}^{1 / 4}\left(\alpha_{n}\right) \operatorname{det}^{-1 / 2}\left(\frac{\alpha_{n}+1}{2}\right) \\
& =\operatorname{det}^{-1 / 4}\left(\frac{h_{n}+h_{n}^{-1}}{2}\right)^{2}=\operatorname{det}^{-1 / 4}\left[1+\frac{1}{4}\left(h_{n}-h_{n}^{-1}\right)^{2}\right] .
\end{aligned}
$$

Take the limit $n \rightarrow \infty$. Since $\Omega_{s} \in \mathscr{F}$ and $h-h^{-1}$ is H. S., this is nonvanishing finite. (Moreover this is positive since $\operatorname{det}(A)>0$ if $A$ is symmetric $\varphi$-selfadjoint operator such that $\left.A_{r}>0\right)$.

If $T_{B}=V_{1} S V_{2}$ with $V_{i} \varphi$-unitary, then

$$
V_{2}^{-1}\left(\frac{h+h^{-1}}{2}\right)^{2} V_{2}=B_{+}^{(\varphi)} B_{+}=1+B_{-}^{(\varphi)} B_{-} .
$$

For the above $T_{B}, U_{B}=\Gamma\left(V_{1}\right) U_{S} \Gamma\left(V_{2}\right)$. Since $\left\{\Gamma\left(V_{i}\right)\right\}$ do not change the Fock vacuum, if $\Omega_{s} \in D\left(\Gamma\left(V_{2}^{-1}\right)\right)$, then

$$
\left\langle\Omega, \Omega_{B}\right\rangle=\left\langle\Omega, \Omega_{S}\right\rangle
$$

Q. E. D.

Proof of Theorem 16.

The number operator $N$ is given by

$$
\sum_{i} a^{*}\left(e_{i}\right) a\left(e_{i}\right)=\frac{1}{2} \sum_{i}\left(-\frac{\partial}{\partial q_{i}}+2 q_{i}\right) \frac{\partial}{\partial q_{i}}
$$

in the $Q-$ space. Let

$$
\Omega_{S}^{\prime} \equiv N \Omega_{S}=\sum_{i} \Omega_{S}^{(i)}
$$

where

$$
\Omega_{s}^{(i)}=\frac{1}{2}\left[\left(\alpha_{i i}-1\right)+q_{i}^{2}-\left(\sum_{j} \alpha_{i j} q_{j}\right)^{2}\right] \Omega_{s} \equiv f_{i}(q) \Omega_{s} .
$$

Since $\alpha-1 \in \mathscr{C}_{2}$ and $\alpha^{-1}$ is bounded, it is explicitly shown that

$$
\left\|\Omega_{s}^{\prime}\right\|_{\mid 2}^{2}=\sum_{i, j} \int \bar{f}_{i} f_{j}\left|\Omega_{S}\right|^{2} d \mu_{0}<\infty \text {. }
$$

Q. E. D.

Remark 6. By similar method, it will be proved that $\Omega_{s} \in D\left(N^{n}\right)$ for $n=1,2, \cdots$. This is the "locally Fock property" of the vacuum defined by Glimm and Jaffe $[7,8]$. 
Finally in this section, we prove that the condition spec $\alpha_{r}>0$ is necessary to ensure $\Omega_{s} \in \mathscr{F}$.

Theorem 27. Let $S$ be a generalized $\varphi$-scaling. The vector $\Omega_{s}$ such that

$$
\left\langle\Omega, \pi_{s}(P) \Omega\right\rangle=\left\langle\Omega_{s}, P \Omega_{s}\right\rangle
$$

is not in the Fock space if inf spec $\alpha_{r}<0$.

Proof. $\alpha=h^{-2}$ takes the following form on $J K_{+} \oplus J K_{-}=J K$ due to the $\varphi$-selfadjointness of $\alpha$ :

$$
\left(\begin{array}{ll}
\left(\alpha_{r}\right)_{++} & \left(\alpha_{i}\right)_{+-} \\
\left(\alpha_{i}\right)_{-+} & \left(\alpha_{r}\right)_{--}
\end{array}\right),
$$

namely $\alpha_{r}=\left(\alpha_{r}\right)_{++} \oplus\left(\alpha_{r}\right)_{--}$on $J K_{+} \oplus J K_{-}$. First assume that inf spec $\left(\alpha_{r}\right)_{++}=-\lambda<0$. Then there is an eigenvector $f \in J K_{+}$of $\alpha_{r}$ belonging to the eigenvalue $-\lambda$. In this case $\Phi_{\varphi}(f)$ is selfadjoint and $\left\|\exp i \Phi_{\varphi}(f)\right\|=1$. Now

$$
\begin{aligned}
& \left\langle\Omega, \pi_{S}\left[\exp \left(i \Phi_{\varphi}(f)\right)\right] \Omega\right\rangle=\left\langle\Omega, \exp \left(i \Phi_{\varphi}(S f)\right) \Omega\right\rangle \\
& =\exp \left[-\frac{1}{4}\langle S f, S f\rangle\right]=\exp \left[-\frac{1}{4}\left(f,\left(\alpha_{r}\right)_{++} f\right)\right] \\
& =\exp \left[\frac{\lambda}{4}(f, f)\right] .
\end{aligned}
$$

If $\lambda>0$, then the right hand side can be made arbitralily large, which contradicts $\mid\left\langle\Omega_{s}\right.$, $\left.\exp \left(i \Phi_{\varphi}(f)\right) \Omega_{s}\right\rangle|\leqq|\left|\Omega_{s}\right|_{2}^{2}<\infty$.

The case of inf $\operatorname{spec}\left(\alpha_{r}\right)_{--}<0$ is similarly discussed. Q. E. D.

We remark that the state $\rho_{B}=\left\langle\Omega, \pi_{B}(.) \Omega\right\rangle$ cannot be extended in general to a state on the $C^{*}$-a gebra generated by $\exp i \Phi(f)$; $f \in \mathscr{H}\}$, as can be immediately seen from the above proof.

\section{§11. Examples of @-unitary Quasi-Implementability}

For weakly $\Theta$-unitary implementability of a Bogolyubov transformation $B$, we obtained the following sufficient condition in 
Theorem 13 :

$$
S_{-} \in \text { H. S., and } \operatorname{spec}(\alpha)_{r}>0
$$

and the following necessary condition in Theorems 12 and 27 :

$$
S_{-} \in \text { H. S., and } \operatorname{spec}(\alpha)_{r} \geqq 0 \text {. }
$$

We now want to present two examples of $B$ where this necessary condition is not satisfied and yet a quantity which can be interpreted to be the overlap $\left\langle\Omega, \Omega_{B}\right\rangle$ is-non vanishing finite in contrast to the situation of positive definite metric where the last condition is equivalent to the unitary implementability [Remark 5].

The first simple example provides the case where $B_{-}$is $\mathrm{H}$. S. but spec $\alpha_{r}$ can become negative. The second example provides a case with $B_{-}$non H.S.

Let $\left\{\mathscr{H}_{l} ; l=1,2, \cdots\right\}$ be a sequence of two-dimensional Hilbert space $\mathscr{H}_{l}=C^{2}$ equipped with an indefinite sesquilinear form $\langle\rangle=$, $\left(, \varphi_{l}\right)$ where $\varphi_{l}=\operatorname{diag}(1,-1)$. Let $\mathscr{H}=\oplus_{l} \mathscr{H}_{l}$ be a Hibelt space equipped with an indefinite sesquilinear form $\langle\rangle=,(, \varphi), \varphi=\oplus_{l} \varphi_{l}$. Let $\left\{T^{(l)} ; l=1,2, \cdots\right\}$ be a sequence of $\varphi$-symplectic operator on $\mathscr{H}_{l}$, and let $\left\{\mathscr{F}_{l},\langle\rangle,\right\}$ be a Fock space equipped with an indefinite sesquilinear form $\langle,\rangle \equiv\left(, \Gamma_{l}\left(\varphi_{l}\right)\right)$ constructed from $\left\{\mathscr{H}_{l},\langle\rangle,\right\}$ with the Fock vacuum $\Omega_{l}$. The Fock space over $\mathscr{H}$ is identified with

$$
\mathscr{F}=\otimes_{l}\left(\mathscr{F}_{l}, \Omega_{l}\right), \Theta=\Gamma(\varphi)=\bigotimes_{l} \Gamma_{l}\left(\varphi_{l}\right)
$$

\section{Example 1.}

$$
T^{(l)}=\left(\begin{array}{lr}
h_{l} & 0 \\
0 & h_{l}^{-1}
\end{array}\right)
$$

on $K_{l} \oplus J K_{l}$, where $K_{l}=K_{+}^{l} \oplus K_{-}^{l}$ and $h_{l}$ is such that the corresponding matrix $\alpha_{i}$, is given by

$$
\alpha_{l}=\lambda_{l}^{2}\left(\begin{array}{ll}
\cos 2 \theta_{l} & i \sin 2 \theta_{l} \\
i \sin 2 \theta_{l} & \cos 2 \theta_{l}
\end{array}\right) \equiv \lambda_{l}^{2} u\left(2 \theta_{l}\right)
$$

with $0 \leqq \theta_{l} \leqq \pi / 2,0<\lambda_{l}$ for all $l$.

The formal vacuum $\Omega_{T}^{(l)}$ is given by 


$$
\begin{aligned}
& {\left[\operatorname{det}\left(\alpha_{l}\right)\right]^{1 / 4} \exp \left[-\frac{1}{2}\left(\mathrm{q}^{(l)},\left(\alpha_{l}-1\right) q^{(l)}\right)\right]} \\
& =\lambda_{l} \exp \left[-\frac{1}{2}\left(q^{(l)},\left(\lambda_{l}^{2} u\left(2 \theta_{l}\right)-1\right) q^{(l)}\right)\right]
\end{aligned}
$$

where

$$
q^{(l)}=\left(\begin{array}{c}
q_{1}^{(l)} \\
q_{2}^{(l)}
\end{array}\right)
$$

Then $\Omega_{T}^{(l)} \in \mathscr{F}_{l}$ only for $0 \leqq 2 \theta_{l}<\pi / 2$ and

$$
\left\|\Omega_{T}^{(l)}\right\|_{p}^{p}=\left[\cos 2 \theta_{l}\right]^{-p / 2} \frac{x_{l}^{p}}{1+\frac{p}{2}\left(x_{l}^{2}-1\right)}
$$

with

$$
x_{l}=\lambda_{l}\left(\cos 2 \theta_{l}\right)^{1 / 2}
$$

for $0 \leqq p<2(1-c)^{-1}\left(c=\cos 2 \theta_{l} \times \min \left\{\lambda_{l}^{2}, \lambda_{l}^{-2}\right\}\right)$. The transformed total vacuum is given by

$$
\otimes_{l} \Omega_{T}^{(l)}
$$

$T_{-}=\oplus_{l} T_{-}^{(l)}$ is Hilbert-Schmidt if and only if

$$
\begin{aligned}
& \sum_{l}\left[\cosh ^{2} \tau_{l} \sin ^{2} \theta_{l}+\sinh ^{2} \tau_{l} \cos ^{2} \theta_{l}\right]<\infty \\
\leftrightarrow & \sum_{l}\left|z_{l}-z_{l}^{-1}\right|^{2}<\infty \leftrightarrow \sum_{l}\left|z_{l}-1\right|^{2}<\infty
\end{aligned}
$$

where $\lambda_{l}=\exp \left(\tau_{l}\right)$ and $z_{l}=\lambda_{l} \exp \left(i \theta_{l}\right) . \quad z_{l}$ is one of the eigenvalues of $h_{l}$. $T$ is weakly $\Theta$-unitarily implementable if $0 \leqq \theta_{l}<\pi / 4$ for all $l$ and $T_{-} \in \mathrm{H}$. S.

The overlapping of the vacua is

$$
\left\langle\Omega^{(l)}, \Omega_{T}^{(l)}\right\rangle=\left[\operatorname{det}\left(\frac{h_{l}+h_{l}^{-1}}{2}\right)\right]^{-1 / 2}=2\left|z_{l}+z_{l}^{-1}\right|^{-1}
$$

where $\Omega^{(l)}$ is the Fock vacuum in $\mathscr{F}_{l}$. The formally defined overlapping $\Pi_{l} 2\left|z_{l}+z_{l}^{-1}\right|^{-1}$ can converge to a non-vanishing finite quantity even if spec $\alpha_{r}>0$ does not hold. In this example, the overlapping is non-vanishing if and only if $T_{-} \in \mathrm{H}$. S.

Example 2.

$$
T^{(l)}=\left(\begin{array}{rr}
h_{l} & 0 \\
0 & h_{l}^{-1}
\end{array}\right)
$$


on $K_{l} \oplus J K_{l}$, where on $K_{l}=K_{+}^{l} \oplus K_{-}^{l}$

$$
h_{l}=1+n_{l}\left(\begin{array}{rr}
1 & 1 \\
-1 & -1
\end{array}\right), n_{l} \in R
$$

Note that $h_{l}-1=n_{l}\left(\begin{array}{rr}1 & 1 \\ -1 & -1\end{array}\right)$ is a $\varphi$-selfadjoint nilpotent operator, and

$$
\begin{aligned}
& h_{l}^{-1}=1-n_{l}\left(\begin{array}{rr}
1 & 1 \\
-1 & -1
\end{array}\right), \\
& \alpha_{l}=1-2 n_{l}\left(\begin{array}{rr}
1 & i \\
i & -1
\end{array}\right) .
\end{aligned}
$$

Thus $T_{-}$is H. S. if and only if $\sum_{l}\left|n_{l}^{2}\right|<\infty$, and $T$ is weakly $\Theta_{-}$ unitarily implementable if $T_{-} \in \mathrm{H}$. S. and $\left|n_{l}\right|<1 / 2$ for all $l$. But the overlapping is independent of $\left\{n_{l}\right\}$ :

$$
\left\langle\Omega^{(l)}, \Omega_{T}^{(l)}\right\rangle=1,\left\langle\Omega, \Omega_{T}\right\rangle=1
$$

\section{§12. Applications to Physics}

As an illustration, we consider, in this section, a mass-shift model of vactor field $A_{\mu}$ in two-dimensional space-time with periodic boundary condition (other more non-trivial models, see $[5,11-13,19,29]$ ).

We shall consider Bogolyubov transformations related to this model and discuss its implementabilities. First we consider the Bogolyubov transformations which diagonalize the Hamiltonian of this model and second we consider the time-translation operator of this model as Bogolyubov transformations.

The (Stückelberg) vector field $A_{\mu}$ in two-dimensional space-time can be described in terms of a scalar field $\phi$ on the Fock space $\mathscr{F}_{U}$ with a positive definite inner product and another scalar field $B$ on the Fock space $\mathscr{F}_{B}$ with an indefinite inner product:

$$
A_{\mu}=\frac{1}{\mu}\left[\varepsilon_{\mu \nu} \partial^{\nu} \phi+\partial_{\mu} B\right]
$$

where $\mu>0$ is a mass of $\phi, \varepsilon_{\mu \nu}$ is the antisymmetric tensor with values \pm 1 , and we assume $B$ has a mass $\mu^{\prime} \geqq 0$. $\left(U_{\mu}=\frac{1}{\mu} \varepsilon_{\mu \nu} \partial^{\nu} \phi\right.$ is the Proca 
field of mass $\mu$ in two dimensions and $B$ is the gaugeon field of mass $\mu^{\prime}$. See $[9,11-13]$.)

The total Hamiltonian $H$ of the mass-shift model is then

$$
H=H_{0}(\phi)+H_{0}(B)-\frac{1}{2} \delta \mu^{2} \int: A_{0}^{2}(x, 0)-A_{1}^{2}(x, 0): d x
$$

where $H_{0}(\phi)$ and $H_{0}(B)$ are the free Hamiltonians and: : denotes the Wick product. To set up simple well defined model, we limit the space to the finite interval $\Lambda=[-L / 2, L / 2] \subset R$ with periodic boundary condition and furthermore use the cutoff (periodic) field $A_{\mu, \sigma}(x, 0)$ instead of $A_{\mu}(x, 0)$ (high momentum parts $(|p|>\sigma)$ and zero momentum parts are completely omitted in $A_{\mu, \sigma}(x, 0)$. See [11-13] for details).

Without loss of generality, we set $\mu^{\prime}=0$ in the following, which corresponds to the Landau gauge formalism of the vector field [9, $11-13,19,29]$.

In terms of creation and annihilation operators $\left\{a^{+}(p), a(p), b^{+}(p)\right.$, $b(p) ; p \in \Gamma \equiv 2 \pi Z / L\}$ of $\phi$ and $B$, the Hamiltonian is

$$
H=H(L, \sigma)=\frac{2 \pi}{L} \sum_{p \in \Gamma, \sigma \geqq p>0}: \mathscr{A}^{+}(p) \mathscr{H}(p) \mathscr{A}(p):-E(L, \sigma)
$$

where

$$
\begin{aligned}
E(L, \sigma) & =\sum_{p \in \Gamma, \sigma \geqq p>0}\left(q_{0}-p_{0}-\frac{\delta \mu^{2}}{2 p_{0}}\right) \\
p_{0} & =\left(p^{2}+\mu^{2}\right)^{1 / 2}, \quad q_{0}=\left(p_{0}^{2}+\delta \mu^{2}\right)^{1 / 2}
\end{aligned}
$$

is a constant (the vacuum energy) chosen so that inf $\operatorname{spec} H(L, \sigma)$ $=0$,

$$
\mathscr{A}(p)=\left(\begin{array}{l}
a(p) \\
b(p) \\
a^{+}(-p) \\
b^{+}(-p)
\end{array}\right), \mathscr{A}^{+}(p)=\left(a^{+}(p), b^{+}(p), a(-p), b(-p)\right)
$$

and $\mathscr{H}(p)$ is a $4 \times 4$ hermitian matrix given by

$$
\left(\begin{array}{ll}
\mathscr{H}_{+}(p) & \mathscr{H}_{-}(p) \\
\overline{\mathscr{H}}_{-}(-p) & \overline{\mathscr{H}}_{-}(-p)
\end{array}\right)
$$


with

$$
\begin{aligned}
& \mathscr{H}_{+}(p)=\left(\begin{array}{cc}
p^{0}+\frac{\delta \mu^{2}}{2 p^{0}} & i \frac{\delta \mu^{2}}{\mu^{2}} \omega^{\prime}(p) \\
-i \frac{\delta \mu^{2}}{\mu^{2}} \omega^{\prime}(p) & -|p|
\end{array}\right) \\
& \mathscr{H}_{-}(p)=\left(\begin{array}{cc}
p^{0} \frac{\delta \mu^{2}}{\mu^{2}}-\frac{\delta \mu^{2}}{2 p^{0}} & i \frac{\delta \mu^{2}}{\mu^{2}} \omega(p) \\
-i \frac{\delta \mu^{2}}{\mu^{2}} \omega(p) & \delta \mu^{2} \\
\mu^{2} & \mu^{2} \mid
\end{array}\right)
\end{aligned}
$$

where

$$
\omega(p)=\frac{\left(p^{0}+|p|\right) p}{2 \sqrt{p^{0}|p|}}, \omega^{\prime}(p)=\frac{\left(p^{0}-|p|\right) p}{2 \sqrt{p^{0}|p|}} .
$$

In this approximation, $\mathscr{H}_{+}=\mathscr{H}_{-}=l^{2}(\Gamma)$ and $\mathscr{F}_{U}=\mathscr{F}_{(}\left(\mathscr{H}_{+}\right), \mathscr{F}_{B}=$ $\mathscr{F}\left(\mathscr{H}_{-}\right)$and the following CCR with an indefinite metric hold:

$$
\left\{\begin{array}{l}
{\left[a^{+}(p), a(q)\right]=-\left[b^{+}(p), b(q)\right]=\frac{L}{2 \pi} \delta_{p, q},} \\
\text { any other commutator }=0 .
\end{array}\right.
$$

Here

$$
a^{+}(p)=[a(p)]^{(\theta)}=[a(p)]^{*}, b^{+}(p)=[b(p)]^{(\theta)}=-[b(p)]^{*},
$$

and $\{a(p), b(p) ; p \in \Gamma\}$ are the annihilation operators:

$$
a(p) \Omega=b(p) \Omega=0
$$

for all $p$.

We shall now discuss the following items about the Hamiltonian (12-1) :

( I ) Diagonalization of $H=H(L, \sigma)$ by a $\varphi$-symplectic operator $T_{\sigma}$ in the sense which shall be explained.

(II) The implementability of $T_{\sigma}$.

(III) The Bogolyubov transformation $\alpha_{t}\left(=\exp \left[i t \delta_{H}\right]\right)$ induced by $H$.

(IV) The implementability of $\alpha_{t}$.

Before studying these problems, we shall note a property of $H(L, \sigma)$, namely $H(L, \sigma)$ is $\Theta_{\text {-symmetric : }}$

$$
\langle\phi, H(L, \sigma) \phi\rangle=\langle H(L, \sigma) \phi, \phi\rangle \text { for } \phi, \phi \in D(H(L, \sigma)) .
$$

Further in the present case $H(L, \sigma)$ is $\Theta$-selfadjoint. In fact the 
symmetric operator $\Theta H(L, \sigma)$ is selfadjoint since $\sigma<\infty$, which implies that $H(L, \sigma)$ is $\Theta$-selfadjoint $[5,13]$.

I and II: We choose a $4 \times 4$ matrix $S(p)$ which diagonalizes $\mathscr{H}(p)$ and leaves the CCR (with indefinite metric) invariant:

$$
\begin{aligned}
& S^{*}(p) \mathscr{H}(p) S(p)=\text { diagonal matrix, } \\
& S^{*}(p) T S(p)=S(p) T S^{*}(p)=T,
\end{aligned}
$$

where

$$
T=\left(\begin{array}{rr}
\varphi & 0 \\
0 & -\varphi
\end{array}\right)
$$

with $\varphi=\operatorname{diag}(1,-1)$. This $S(p)$ is obtained by solving the following equations :

$$
\operatorname{det}[\mathscr{H}(p)-T x]=0, \mathscr{H}(p) T u_{i}=e_{\imath} u_{\imath}
$$

with the normalization with respect to $(, T)=\langle,\rangle_{T}$ :

$$
\left\langle u_{\imath}, u_{j}\right\rangle_{T}=(T)_{\imath} \text {. }
$$

Here $\left\{e_{i} ; i=1, \cdots, 4\right\}$ are the roots of the characteristic equation, and are given by $\left\{ \pm q^{0}= \pm\left(p^{2}+\mu^{2}+\delta \mu^{2}\right)^{1 / 2}, \pm|p|\right\}$. Then $S(p)_{i j}=$ $\left(T u_{j}(p)\right)_{i}$ :

$$
S(p)=\left(\begin{array}{ll}
\frac{S_{+}(p)}{S_{-}(-p)} & \frac{S_{-}(p)}{S_{+}(-p)}
\end{array}\right)
$$

where

$$
\begin{aligned}
& S_{+}(p)=\left(\begin{array}{cc}
\frac{\bar{\mu}^{2} p^{0}+\mu^{2} q^{0}}{2 \mu \bar{\mu} \sqrt{p^{0} q^{0}}} & -\frac{i \delta \mu^{2} p}{2 \mu \bar{\mu} \sqrt{p^{0}|p|}} \\
\frac{i \delta \mu^{2} p}{2 \mu \bar{\mu} \sqrt{q^{0}|p|}} & \frac{\mu^{2}+\bar{\mu}^{2}}{2 \mu \bar{\mu}}
\end{array}\right) \\
& S_{-}(p)=\left(\begin{array}{cc}
\frac{\mu^{2} q^{0}-\bar{\mu}^{2} p^{0}}{2 \mu \bar{\mu} \sqrt{p^{0} q^{0}}} & -\frac{i \delta \mu^{2} p}{2 \mu \bar{\mu} \sqrt{p^{0}|p|}} \\
-\frac{i \delta \mu^{2} p}{2 \mu \bar{\mu} \sqrt{q^{0}|p|}} & \frac{\delta \mu^{2}}{2 \mu \bar{\mu}}
\end{array}\right)
\end{aligned}
$$

with $\bar{\mu}=\left(\mu^{2}+\delta \mu^{2}\right)^{1 / 2}$.

Let

$$
B_{+}(p)=\left(\begin{array}{cc}
S_{+}(p) & 0 \\
0 & S_{+}(-p)
\end{array}\right)^{*}, B_{-}(p)=C\left(\begin{array}{cc}
0 & S_{-}(p) \\
S_{-}(-p) & 0
\end{array}\right)^{*},
$$


where $C$ is the complex conjugation operator on $\mathscr{H}$. Let

$$
T(p)=B_{+}(p)+B_{-}(p),
$$

and let

$$
B_{ \pm}(\sigma)=\bigoplus_{0<p \leqq o} B_{ \pm}(p), T_{\sigma}=B_{+}(\sigma)+B_{-}(\sigma) .
$$

Thus $U_{T_{\sigma}}$ should implement $\mathscr{A}(p) \rightarrow S(p) \mathscr{A}(p)$, and then implement $A_{\mu, \sigma}(x, 0) \rightarrow \tilde{A}_{\mu, \sigma}(x, 0)$, where $\tilde{A}_{\mu, \sigma}(x, 0)$ is the vector field of mass $\bar{\mu}=\left(\mu^{2}+\delta \mu^{2}\right)^{1 / 2}$ (in the Landau gauge formalism).

Since $\varphi S_{ \pm}(-p) \varphi=S_{ \pm}(p)$ in this case, we see $U_{0} T_{\sigma} U_{0}^{-1}=\widetilde{T}_{\sigma}$ where

$$
U_{0}=\frac{1}{\sqrt{2}}\left(\begin{array}{cc}
1 & \varphi \\
-\varphi & 1
\end{array}\right)
$$

is a unitary matrix commuting with $\varphi$, and

$$
\widetilde{T}_{\sigma}=\left(\begin{array}{lr}
t_{\sigma}(+) & 0 \\
0 & t_{\sigma}(-)
\end{array}\right),
$$

where $t_{o}( \pm)=\bigoplus_{0<p \leqq o} t( \pm p)$ with

$$
t( \pm p)=S_{+}^{*}( \pm p) \pm \varphi C S_{-}^{*}( \pm p) .
$$

Thus it suffices to consider the implementability of $\widetilde{T}_{\sigma}$. Since for $K_{\varphi}=K_{+} \oplus i K_{-}$,

$$
\begin{aligned}
& t_{\sigma}( \pm) K_{\varphi} \subset K_{\varphi}, t_{\sigma}( \pm) \vdash K_{\varphi}=\bigoplus_{0<p \leqq o}\left(S_{+}^{*}( \pm p) \pm S_{-}^{*}( \pm p)\right) \equiv t_{\sigma}( \pm)_{1}, \\
& t_{\sigma}( \pm) J K_{\varphi} \subset J K_{\varphi}, t_{\sigma}( \pm) \uparrow J K_{\varphi}=\bigoplus_{0<p \leqq v}\left(S_{+}^{*}( \pm p) \mp S_{-}^{*}( \pm p)\right) \equiv t_{\sigma}( \pm)_{2},
\end{aligned}
$$

$\widetilde{T}_{\sigma}$ takes the following form on $K_{\varphi} \oplus J K_{\varphi}$ :

$$
\left(\begin{array}{ll}
T_{\sigma, 1} & 0 \\
0 & T_{\sigma, 2}
\end{array}\right)
$$

where

$$
T_{\sigma, 1}=\left(\begin{array}{cc}
t_{\sigma}(+)_{1} & 0 \\
0 & t_{\sigma}(-)_{1}
\end{array}\right), \quad T_{\sigma, 2}=\left(\begin{array}{ll}
t_{\sigma}(+)_{2} & 0 \\
0 & t_{\sigma}(-)_{2}
\end{array}\right)
$$

and, of course, $J T_{\sigma .1}^{-1} J^{-1}=T_{\sigma .2}^{(\varphi)}$.

We investigate $\alpha \equiv T_{\sigma, 2}^{(\varphi)} T_{\sigma, 2}$ which is $\varphi$-selfadjont and symmetric:

$$
\alpha=\bigoplus_{-\sigma \leqq p \leqq \sigma, p \neq 0} \alpha(p)
$$




$$
\begin{aligned}
\alpha(p) & =\left(S_{+}^{*}(p)-S_{-}^{*}(p)\right)^{(\varphi)}\left(S_{+}^{*}(p)-S_{-}^{*}(p)\right) \\
& =\left(\begin{array}{ll}
\left(\frac{\bar{\mu}}{\mu}\right)^{2}-\frac{p^{0}}{q^{0}} & i \frac{\delta \mu^{2}}{\mu^{2}} \frac{p^{0} p}{q^{0} \sqrt{p^{0} p}} \\
i \frac{\delta \mu^{2}}{\mu^{2}} \frac{p^{0} p}{q^{0} \sqrt{p^{0} p}} & \frac{1}{\mu^{2} \bar{\mu}^{2}}\left[\mu^{4}-\frac{p}{q^{0}}\left(\delta \mu^{2}\right)^{2}\right]
\end{array}\right) \\
\alpha(-p) & =\left(S_{+}^{*}(-p)+S_{-}^{*}(-p)\right)^{(\varphi)}\left(S_{+}^{*}(-p)+S_{-}^{*}(-p)\right) \\
& =\left(\begin{array}{ll}
\frac{q^{0}}{\left.\mu^{2} \frac{\mu^{2} p^{0}}{\mu^{4}-\frac{p}{q^{0}}}\left(\delta \mu^{2}\right)^{2}\right]} & i \frac{\delta \mu^{2}}{\mu^{2}} \frac{p}{\sqrt{p^{0} p}} \\
i \frac{\delta \mu^{2}}{\mu^{2}} \frac{p}{\sqrt{p^{0} p}} & \left(\frac{\bar{\mu}}{\mu}\right)^{2}
\end{array}\right)
\end{aligned}
$$

with $p>0$. Therefore $\alpha_{r}(p)>0$ for all $p$ if $\left|\delta \mu^{2}\right|<\mu^{2}$, and

$$
\lim _{p \rightarrow \pm \infty} \alpha(p)=1+\frac{\delta \mu^{2}}{\mu^{2}}\left(\begin{array}{ll} 
\pm 1 & i \\
i & \mp 1
\end{array}\right)+O\left(p^{-2}\right) .
$$

The formal vacuum $\Omega_{B}$ is given by $[\operatorname{det}(\alpha)]^{1 / 4} \exp \left[-\frac{1}{2}(q,(\alpha-1) q)\right]$ which is in the Fock space if $\sigma<\infty$ and $\left|\delta \mu^{2}\right|<\mu^{2}$.

The overlapping is given by

$$
\operatorname{det}^{-1 / 2}\left[B_{+}(\sigma)\right] \equiv \omega_{0}(L, \sigma)=\Pi_{0<p \leqq o} \operatorname{det}^{-1} S_{+}(p)
$$

and is non-vanishing finite for $\delta \mu^{2} \geqq-\mu^{2}$, and absolutely converges to a non-vanishing finite value $\omega_{0}(L, \infty)$ as $\sigma \rightarrow \infty$ since

$$
\begin{aligned}
\operatorname{det}^{-1} S_{+}(p) & =\frac{4 \mu^{2} \bar{\mu}^{2} \sqrt{p^{0} q^{0}}}{\left(\mu^{2}+\bar{\mu}^{2}\right)\left(\bar{\mu}^{2} p^{0}+\mu^{2} q^{0}\right)-\left(\delta \mu^{2}\right)^{2}|p|} \\
& =1+O\left(p^{-2}\right)
\end{aligned}
$$

as $p \rightarrow \infty$.

As conclusions, $T_{\sigma}$ is weakly $\Theta$-unitarily implementable for $\sigma<\infty$ and $\left|\delta \mu^{2}\right|<\mu^{2}$, and $\Theta$-unitarily quasi-implementable for $\sigma \leqq \infty$ and $\delta \mu^{2} \geqq-\mu^{2}$.

Remarks 7. (1) The following quantity exists:

$$
\alpha_{p} \equiv \lim _{L, \sigma \rightarrow \infty}-\frac{1}{L}-\log \omega_{0}(L, \sigma)=\frac{1}{2 \pi} \int_{0}^{\infty} d p \log \left[\operatorname{det}\left(S_{+}(p)\right)\right] .
$$

This is called the effective potential in field theory (the subscript " $p$ " means the periodic boundary condition). Since the indefinite metric formalism is used, this is not necessarily positive. In fact $\operatorname{det}\left(S_{+}(p)\right)<1$ 
for large $|p|$.

(2) The vacuum energy per unit volume converges for $L, \sigma \rightarrow \infty$ :

$$
\lim _{L, \sigma \rightarrow \infty} \frac{1}{L} E(L, \sigma)=\frac{1}{2 \pi} \int_{0}^{\infty} d p\left(q^{0}-p^{0}-\frac{\delta \mu^{2}}{2 p^{0}}\right) .
$$

(3) Let $\Phi_{\varphi}(f)$ be the $\Theta$-selfadjoint field as before. Then

$$
\begin{aligned}
\rho_{B}\left(\exp i \Phi_{\varphi}(f)\right) & =\left\langle\Omega, \exp \left(i \Phi_{\varphi}(B f)\right) \Omega\right\rangle \\
& =\exp \left[-\frac{1}{4}\langle B f, B f\rangle\right]
\end{aligned}
$$

which converges uniformly as $L, \sigma \rightarrow \infty$ for any $f \in L^{2}\left(R^{2} ; d^{2} x\right)$. (See also $[7,8,22,23]$.)

III and IV: Let $H_{0}(L, \sigma)$ be the diagonalized Hamiltonian by the transformation " $S(p)$ ", and let $U_{T}$ be the operator on the Fock space which implements the transformation of the fields. Since $U_{T}$ is unbounded, we can say that $H(L, \sigma)$ is similar to the selfadjoint operator $H_{0}(L, \sigma)$ which commutes with $\Theta$ by unbounded $\Theta$-isometric operator $U_{T^{*}}$. By the definition of $E(L, \sigma)$, inf $\operatorname{spec} H_{0}(L, \sigma)=0$.

We say that a closed linear subspace $\mathscr{S}$ in $\mathscr{H}$ is uniformly definite (resp. strongly definite) if it is uniformly positive or negative (resp. positive or negative). We have obtained the spectral resolution of $H(L, \sigma)$ in terms of $\Theta$-selfadjoint projection operators (for $\left|\delta \mu^{2}\right|$ $\left\langle\mu^{2}\right)$. But their ranges are not uniformly definite.

$H(L, \sigma)$ is considered to be the generator of the following differential equation:

$$
\begin{aligned}
i \frac{d}{d t} \phi(t) & =H(L, \sigma) \phi(t), \\
\phi(t=0) & =\phi_{0} \in \mathscr{F}
\end{aligned}
$$

i. e., we formally have

$$
\phi(t)=U(-\mathrm{t}) \phi_{0}, U(t)=\exp [i t H(L, \sigma)] .
$$

We proved that $H(L, \sigma)$ is $\Theta$-selfadjoint, but formally defined $U( \pm t)$ cannot be bounded in general except for $t=0: U(t)$ is an unbounded operator which implements the following transformation of the field: 


$$
\alpha_{t}\left(\Phi_{\varphi}(f)\right) \equiv \exp i t \delta_{H} \cdot \Phi_{\varphi}(f)=\Phi_{\varphi}(B(t) f),
$$

where $B(t)=B^{-1} K_{0}(t) B=B_{+}(t)+B_{-}(t)$ is a one-parameter group of $\varphi$-symplectic operator and $K_{0}(t)$ is a one-parameter unitary group defined by

$$
\exp i t \delta_{H 0} \cdot \Phi_{\varphi}(f)=\Phi_{\varphi}\left(K_{0}(t) f\right)
$$

$B_{ \pm}(t)$ are explicitly given as follows :

$$
\left\{\begin{array}{l}
B_{+}(t)=B_{+}^{(\varphi)} K_{0}(t) B_{+}-B_{-}^{(\varphi)} K_{0}(t) B_{-}, \\
B_{-}(t)=B_{+}^{(\varphi)} K_{0}(t) B_{-}-B_{-}^{(\varphi)} K_{0}(t) B_{+} .
\end{array}\right.
$$

Even if $\left(B_{+}, B_{-}\right)$is weakly $\Theta$-unitarily implementable, $\left(B_{+}(t)\right.$, $\left.B_{-}(t)\right)$ is not necessarily weakly $\Theta$-unitarily implementable. Such a phenomenon can be easily confirmed in a simpler example. $\left(B_{+}(t)\right.$, $\left.B_{-}(t)\right)$ is $\Theta$-unitarily quasi-implementable in general. (Note that $B_{-}(t)$ is H. S. if so is $B_{-}$.)

Remark 8. One-parameter $\Theta$-unitary group $U(t)$ is called stable [18] if $\|U(t)\| \leqq M$ for all $t \in R$. By connecting results in $[18,21]$, we see that the necessary and sufficient condition for $H$ to be a generator of one-parameter stable $\Theta$-unitary group is that $H$ is similar to a selfadjoint operator $H_{0}$. On the other hand, by a theorem owing to Phillips $[18,21]$, we see that the necessary and sufficient condition for the $\Theta$-selfadjoint operator $H$ to be similar to a selfadjoint operator $H_{0}$ is that $H$ has two closed invariant uniformly definite subspaces $\mathscr{F}^{( \pm)}$such that $\mathscr{F}^{\left({ }^{\prime}\right.} \mathscr{F}^{(+)} \oplus \mathscr{F}^{(-)}$with respect to $\langle$,$\rangle . In$ physics, it is not expected that the Hamiltonian becomes a generator of a one-parameter stable $\Theta$-unitary group.

\section{§13. Concluding Remarks}

We first discuss some properties of $U_{B}^{-1}$ which is weakly $\Theta$ unitarily implemented. For simplicity, we assume that $B$ is $\varphi$-unitary (then $U_{B}^{-1}=\Gamma\left(B^{-1}\right)$ ).

Let $\varphi=P_{+}-P_{-}$as before and let $\mathscr{H}=\mathscr{H}_{+} \oplus \mathscr{H}_{-}$where $\mathscr{H}_{ \pm}=P_{ \pm} \mathscr{H}$. Let $B^{-1} \mathscr{H}=B^{-1} \mathscr{H}_{+} \oplus B^{-1} \mathscr{H}_{-}$. The topology of $\mathscr{H}$ is introduced by 
the inner product

$$
(x, x)_{1} \equiv\left\langle x_{+}, x_{+}\right\rangle-\left\langle x_{-}, x_{-}\right\rangle
$$

where $x_{ \pm} \in \mathscr{H}_{ \pm}$, while the topology of $B^{-1} \mathscr{H}$ is defined by the inner product

$$
(x, x)_{2} \equiv\left\langle x_{+}, x_{+}\right\rangle-\left\langle x_{-}, x_{-}\right\rangle
$$

where $x_{ \pm} \in B^{-1} \mathscr{H}_{ \pm}$. Since $B x_{ \pm} \in \mathscr{H}_{ \pm}$, we have

$$
\begin{aligned}
(x, x)_{2} & =\left\langle B^{-1} B x_{+}, B^{-1} B x_{+}\right\rangle-\left\langle B^{-1} B x_{-}, B^{-1} B x_{-}\right\rangle \\
& =\left\langle B x_{+}, B x_{+}\right\rangle-\left\langle B x_{-}, B x_{-}\right\rangle=\left(B x_{+}, B x_{+}\right)_{1}+\left(B x_{-}, B x_{-}\right)_{1} .
\end{aligned}
$$

Thus there are constants $0<\mu_{1} \leqq \mu_{2}<\infty$ such that

$$
\mu_{1}|| x\left\|_{1}^{2} \leqq\right\| x\left\|_{2}^{2} \leqq \mu_{2}|| x\right\|_{1}^{2}
$$

which means that the topologies of $\mathscr{H}$ and $B^{-1} \mathscr{H}$ are equivalent. The unitary and hermitian operator $\varphi$ is again represented as a unitary and hermitian operator $\tilde{\varphi}$ :

$$
\tilde{\varphi}=P_{+}^{\prime}-P_{-}^{\prime},
$$

where $P_{ \pm}^{\prime}$ are projections to $B^{-1} \mathscr{H}_{ \pm}$and are selfadjoint with respect to $(,)_{2}$.

Next let $\mathscr{F}(\mathscr{H})=\mathscr{F}\left(\mathscr{H}_{+}\right) \otimes \mathscr{F}\left(\mathscr{H}_{-}\right)$and let

$$
\Theta=\Gamma(\varphi)=\Theta_{+}-\Theta_{-}
$$

where $\Theta_{ \pm}$are projections :

$$
\Theta=\left\{\begin{aligned}
1 & \text { on } \mathscr{F}\left(\mathscr{H}_{+}\right) \otimes \mathscr{F}^{(2 n)}\left(\mathscr{H}_{-}\right), \\
-1 & \text { on } \mathscr{F}\left(\mathscr{H}_{+}\right) \otimes \mathscr{F}^{(2 n+1)}\left(\mathscr{H}_{-}\right)
\end{aligned}\right.
$$

where $n=0,1, \cdots$. Let $\mathscr{F}_{ \pm}=\Theta_{ \pm} \mathscr{F}$. Then the topology of the Fock space $\mathscr{F}$ is defined by the inner product

$$
(\phi, \phi)_{1}=\left\langle\phi_{+}, \phi_{+}\right\rangle-\left\langle\phi_{-}, \phi_{-}\right\rangle
$$

where $\phi_{ \pm} \in \mathscr{F}_{ \pm}$, while the topology of $\mathscr{F}\left(B^{-1} \mathscr{H}_{+}\right) \otimes \mathscr{F}\left(B^{-1} \mathscr{H}_{-}\right)$is given by

$$
(\phi, \phi)_{2}=\left\langle\phi_{+}, \phi_{+}\right\rangle-\left\langle\phi_{-}, \phi_{-}\right\rangle
$$

where $\phi_{ \pm} \in \mathscr{F}_{ \pm}\left(B^{-1} \mathscr{H}\right)$ and $\mathscr{F}_{ \pm}\left(B^{-1} \mathscr{H}\right)=\bigoplus_{n=\text { even }(\text { odd })} \mathscr{F}\left(B^{-1} \mathscr{H}_{+}\right) \otimes \mathscr{F}^{(n)}$ $\left(B^{-1} \mathscr{H}_{-}\right)$. 


$$
\left\{\begin{array}{l}
\Gamma^{(n)}\left(B^{-1}\right)=\Gamma\left(B^{-1}\right)\left\lceil\mathscr{F}^{(n)}(\mathscr{H})=B^{-1} \otimes \cdots \otimes B^{-1}(n \text {-times })\right. \\
\mathscr{F}^{(n)}\left(\mathscr{H}^{\prime}\right)=\bigoplus_{n_{+}+n_{-}=n} \mathscr{F}^{\left(n_{+}\right)}\left(\mathscr{H}_{+}\right) \otimes \mathscr{F}^{\left(n_{-}\right)}\left(\mathscr{H}_{-}\right) .
\end{array}\right.
$$

Then for $\phi \in \mathscr{F}^{(n)}\left(B^{-1} \mathscr{H}\right)=\Gamma^{(n)}\left(B^{-1}\right) \mathscr{F}^{(n)}(\mathscr{H})$, we see

$$
\|\phi\|_{2}^{2}=\left\|\Gamma^{(n)}(B) \phi\right\|_{1}^{2} .
$$

Therefore there is a vector $\phi$ in $\mathscr{F}$ such that $\|\phi\|_{1}^{2}<\infty$ and $\|\phi\|_{2}^{2}=\infty$. This shows that $\mathscr{F}(\mathscr{H})$ and $\mathscr{F}\left(B^{-1} \mathscr{H}\right)$ are different.

Due to this phenomenon, the set of weakly $\Theta$-unitarily implementable $B$ does not form a group and is not invariant by $\varphi$-unitary operators.

Remark 9. If $V$ is $\varphi$-unitary, $\Phi_{\varphi}(V f)=\Gamma(V) \Phi_{\varphi}(f) \Gamma\left(V^{-1}\right)$. Then for a decomposition $T=V_{1} S V_{2}$ with $V_{i} \varphi$-unitary, we define $U_{T}=$ $\Gamma\left(V_{1}\right) U_{S} \Gamma\left(V_{2}\right)$. Since $\Gamma(V) \Omega=\Omega, \Omega_{T}=\Omega_{S}$ if $T=V S$ (as in Theorem 14). Contrary to the case of generalized $\varphi$-scalings, even if $\Omega_{T} \in \mathscr{F}$, $\Omega_{T^{-1}}$ is not necessarily in the Fock space.

In this paper, we considered the properties of $\Omega_{B}$ which satisfies

$$
\left\langle\Omega, \pi_{B}(P) \Omega\right\rangle=\left\langle\Omega_{B}, P \Omega_{B}\right\rangle
$$

where $P$ is a polynomial of fields $\left\{\Phi_{\varphi}\left(f_{i}\right)\right\}$ :

$$
P=P\left(\Phi_{\varphi}\left(f_{1}\right), \cdots, \Phi_{\varphi}\left(f_{n}\right)\right),
$$

and

$$
\pi_{B}(P)=P\left(\Phi_{\varphi}\left(B f_{1}\right), \cdots, \Phi_{\varphi}\left(B f_{n}\right)\right)
$$

Let

$$
\rho_{B}(P)=\left\langle\Omega_{B}, P \Omega_{B}\right\rangle .
$$

In physics, expectations $\left\{\rho_{B}(P)\right\}$ are easily calculated rather than $\pi_{B}(P)$ themselves. To obtain $\pi_{B}$ from $\rho_{B}$ is the converse problem which should be investigated in the next step. (See, e. g., [10, 28]. See also $[13,29]$ for the problem in physics.) 


\section{Acknowledgements}

The author would like to thank Professor H. Araki for encouragement and for helpful suggestions. Further this work's starting points are the works of Professors H. Araki and N. Nakanishi. Discussions with his colleagues, Drs. A. Kishimoto, N. Nagamachi and F. Hansen were very helpful. He deeply thanks Dr. S. Ota (Yamagata Univ.) for sending the author his work. He thanks Dr. F. Hansen who kindly considered a problem in this paper with the author.

\section{References}

[1] Araki, H., On the Diagonalization of a Bilinear Hamiltonian by a Bogolyubov Transformation, Publ. RIMS., Kyoto Univ., A-4 (1968), 387.

[2] , On Quasifree States of CAR and Bogolyubov Automorphisms, Publ. RIMS. Kyoto Univ., 6 (1971), 385.

[3] Berezin, F. A., The Method of Second Quantization, Aacdemic Press, N. Y., 1967.

[4] Bognar, J., Indefinite Inner P roduct Space, Springer-Verlag, Berlin 1973.

[5] Bogolyubov, N. N., Shirkov, D. V., Introduction to the Theory of Quantized Field, Interscience Publishers, Inc., N. Y., 1959.

[6] Dunford, N. and Schwartz, J. Y., Linear Operators I, Interscience Publishers, Inc., N. Y., 1967.

[7] Glimm, J. and Jaffe, A., The $\lambda\left(\phi^{4}\right)_{2}$ Quantum Field Theory without Cutoffs (III), Act. Math., 125 (1970), 203.

[8] Statistical Mechanics and Quantum Field Theory, ed. by C. D. Witt et al., Gordon and Breach, N. Y., 1971, 1.

[9] Ginibre, J. and Velo, C., The Free Euclidean Massive Vector Field in the Stückelberg gauge, Ann. Inst. Henri Poincare, 22 (1975), 257.

[10] Hansen, F., RIMS Preprint, in preparation.

[11] Ito, K. R., On the Mechanism by which the Photon Becomes Massive, Nucl. Phys., $102 \mathrm{~B}$ (1976), 309.

[12] - Two-Dimensional QED as a Model in CQFT, Phys. Letters, 65B (1976), 450.

[13] , Construction of (QED) 2 , RIMS-preprint 243 (1977).

[14] Kato, T., Perturbation Theory for Linear Operators, Springer-Verlag, Berlin, 1966.

[15] Klein, A., Quadratic Expressions in a Free Boson Field, Trans. A. M. S., 181 (1973), 439.

[16] Krein, M. G., Plus-Operator in a Space with Indefinite Metric, A. M. S. Transl., 8อ̆ (1970), 93.

[17] Krein, M. G. and Smuljan, J. L., J-polar Representation of Plus-Operators, A. M. S. Transl., $8 \tilde{3}$ (1970), 155.

[18] Krein, M. G., Introduction to the Geometry of Indefinte J-space and to the Theory of Operators in those Spaces, A. M. S. Transl., 93 (1970), 103.

[19] Nakanishi, N., Indefinite Metric Quantum Field Theory, Prog. Theor. Phys. Suppl. Kyoto, $\mathbf{5 1}$ (1972), 1. 
[20] Ota, S., An Operator Algebra in an Indefinite Inner Product Space, Memoirs. Fac. Sci. Kyushu Univ., Ser. A (1975).

[21] Phillips, R. S., In Proc.Int. Sym. Linear Space (Jerusalem Acad. Press, 1960), p. 366.

[22] Rosen, L., Renormalization of the Hilbert Space in the Mass Shift Model, J. Math. Phys., 13 (1972), 918.

[23] Sakai, S., $C^{*}$ algebras and $W^{*}$ algebras, Springer-Verlag, Berlin 1970.

[24] Shale, D., Linear Symmetries of Free Boson Fields, Trans. A. M. S., 103 (1962), 149.

[25] Simon, B. and Seiler, E., An Inequality for Determinants, Proc. Nat. Acad. Sci. To appear.

[26] Simon, B., Note on Infinite Determinants of Hilbert Space Operators, Princeton Univ. Preprint 1975.

[27] , Bogolyubov Transformations, Princeton Univ. Preprint 1972, unpublished.

[28] Tomita, M., In Proceeding of Japan-U. S. Seminar on $C^{*}$ algebra and Applications to Physics, RIMS, Kyoto, 1974.

[29] Wightman, A. S. and Strocchi, F., Proof of Charge Selection Rule in Local Relativistic Quantum Field Theory, J. Math. Phys., 15 (1974), 2189. 